\title{
OPEN APOE-ع4-related differences in left thalamic microstructure in cognitively healthy adults
}

\author{
Jilu P. Mole ${ }^{1}$, Fabrizio Fasano², John Evans ${ }^{1}$, Rebecca Sims ${ }^{3}$, Emma Kidd ${ }^{4}$,
} John P. Aggleton ${ }^{1}$ \& Claudia Metzler-Baddeley ${ }^{1 凶}$

$A P O E-\varepsilon 4$ is a main genetic risk factor for developing late onset Alzheimer's disease (LOAD) and is thought to interact adversely with other risk factors on the brain. However, evidence regarding the impact of $A P O E-\varepsilon 4$ on grey matter structure in asymptomatic individuals remains mixed. Much attention has been devoted to characterising APOE- $\varepsilon 4$-related changes in the hippocampus, but LOAD pathology is known to spread through the whole of the Papez circuit including the limbic thalamus. Here, we tested the impact of $A P O E-\varepsilon 4$ and two other risk factors, a family history of dementia and obesity, on grey matter macro- and microstructure across the whole brain in 165 asymptomatic individuals (38-71 years). Microstructural properties of apparent neurite density and dispersion, free water, myelin and cell metabolism were assessed with Neurite Orientation Density and Dispersion (NODDI) and quantitative magnetization transfer (qMT) imaging. APOE- 4 carriers relative to noncarriers had a lower macromolecular proton fraction (MPF) in the left thalamus. No risk effects were present for cortical thickness, subcortical volume, or NODDI indices. Reduced thalamic MPF may reflect inflammation-related tissue swelling and/or myelin loss in APOE- $\varepsilon 4$. Future prospective studies should investigate the sensitivity and specificity of qMT-based MPF as a non-invasive biomarker for LOAD risk.

As the global population ages, an increasing number of people over 65 will develop dementia due to late onset Alzheimer's disease (LOAD) ${ }^{1}$. LOAD is characterized by the development of amyloid- $\beta$ plaques and neurofibrillary tau tangles that spread from limbic regions to neocortical areas ${ }^{2-4}$. As these pathological processes are thought to accumulate over many years ${ }^{5}$, it may be possible to identify brain changes related to heightened risk in asymptomatic individuals prior to the onset of memory impairment.

Carriage of the Apolipoprotein E (APOE)- $\varepsilon 4$ genotype is the best-established genetic risk factor of LOAD ${ }^{6,7}$. $A P O E$ is the main cholesterol carrier in the brain that supports lipid transport, myelination, synaptic repair and the regulation of amyloid- $\beta$ aggregation and clearance ${ }^{8}$. Individuals who carry the $A P O E-\varepsilon 4$ isoform compared to those with $A P O E-\varepsilon 2$ and $-\varepsilon 3$ show an earlier onset of $\operatorname{LOAD}^{6,9}$ and a larger burden of amyloid- $\beta$ plaques ${ }^{10-14}$. Such harmful effects of $A P O E-\varepsilon 4$ are heightened in individuals with a family history of $\mathrm{LOAD}^{15,16}$, probably due to the presence of other polygenic risk variants such as those of TREM2 $2^{17,18}$. In addition, APOE- 44 is known to combine adversely with lifestyle-related risk notably central obesity ${ }^{19,20}$. Excessive abdominal visceral fat can lead to the metabolic syndrome, type 2 diabetes, and cardiovascular disease ${ }^{21}$ and obese $A P O E-\varepsilon 4$ carriers are more likely to develop hypertension, inflammation and insulin resistance ${ }^{22,23}$.

Much attention has been devoted to characterizing APOE- $\varepsilon 4$-related changes in medial temporal lobe regions, notably in the hippocampus and parahippocampal regions ${ }^{24-26}$ due to their importance for episodic memory. Hippocampal volume loss on magnetic resonance imaging (MRI) is also one of the diagnostic biomarkers of $\mathrm{LOAD}^{27}$. However, hippocampal atrophy is lacking in specificity ${ }^{28}$ and usually occurs in more advanced disease stages $^{29}$. Indeed, evidence regarding hippocampal atrophy in $A P O E$ - $\varepsilon 4$ carriers is mixed and is often thought to result from the inclusion of older participants with underlying LOAD pathology ${ }^{30,31}$. It, therefore, stands to reason that hippocampal volume loss may not be sufficiently sensitive to detect very early disease changes and it has been proposed that focusing on specific hippocampal subregions such as CA1 and subiculum may be

${ }^{1}$ Cardiff University Brain Research Imaging Centre (CUBRIC), School of Psychology, Cardiff University, Maindy Road, Cathays, Cardiff CF24 4HQ, UK. '2Siemens Healthcare, Henkestrasse 127, 91052 Erlangen, Germany. ${ }^{3}$ Division of Psychological Medicine and Clinical Neuroscience, School of Medicine, Cardiff University, Haydn Ellis Building, Maindy Road, Cathays, Cardiff CF24 4HQ, UK. ${ }^{4}$ School of Pharmacy and Pharmaceutical Sciences, Cardiff University, Redwood Building, King Edward VII Avenue „, Cardiff CF10 3NB, UK. ${ }^{凶}$ email: Metzler-BaddeleyC@ cardiff.ac.uk 


\begin{tabular}{|l|l|}
\hline & Mean (SD) (range) \\
\hline Sample size n & 165 \\
\hline Age (in years) & $55.7(8.2)(38-71)$ \\
\hline Females & $57 \%$ \\
\hline NART-IQ & $116.8(6.7)(96-128)$ \\
\hline MMSE & $29.1(0.9)(27-30)$ \\
\hline FH + & $35.8 \%$ \\
\hline APOE4+ & $38.8 \%$ \\
\hline WHR & $1.4(0.5)(0.7-2.2)$ \\
\hline Systolic BP $(\mathrm{mm} \mathrm{Hg})$ & $132(18.8)(68.3-196)$ \\
\hline Diastolic BP $(\mathrm{mm} \mathrm{Hg})$ & $83.3(9.4)(58.7-118.7)$ \\
\hline Smokers & $5.5 \%$ \\
\hline Diabetes & $1.8 \%$ \\
\hline Alcohol units per week & $7.4(9.4)(0-60)$ \\
\hline PHQ-9 Depression score & $2.6(2.9)(0-13)$ \\
\hline
\end{tabular}

Table 1. Summary of demographic, genetic, and lifestyle risk information of CARDS participants. $A P O E=$ Apolipoprotein-E based on DNA extraction and $A P O E$ genotyping of saliva samples using TaqMan genotyping of single nucleotide polymorphism (SNP) rs7412 and KASP genotyping of SNP rs429358. $\mathrm{FH}=$ Family History of a first degree relative affected by Alzheimer's or Lewy body disease or vascular dementia. MMSE $=$ Mini Mental State Exam $(\text { maximum score }=30)^{42}$, NART-IQ $=$ National Adult Reading Test- Intelligence Quotient ${ }^{66}$, PHQ-9 $=$ Patient Health Questionnaire (maximum score $\left.=27\right)^{109}$. WHR $=$ Waistto-Hip-Ratio.

more promising ${ }^{32,33}$. However, it is also possible that limbic regions other than the hippocampus may play an important role in the development of LOAD. Notably, it has been recognised for a while that LOAD pathology may spread through the whole of the Papez circuit and may critically involve the limbic thalamus ${ }^{4}$. For instance, neurofibrillary accumulations in the anterodorsal thalamic nucleus have been found at the same time as those in the hippocampus in LOAD brains ${ }^{34}$ and reduced thalamic MRI volume has been observed in amnestic Mild Cognitive Impairment $(\mathrm{MCI})^{35}, \mathrm{LOAD}^{36}$ and presymptomatic presenilin 1 mutation carriers ${ }^{37}$. Similarly, Positron Emission Tomography (PET) studies have found $A P O E-\varepsilon 4$ state to accelerate longitudinal reductions in glucose metabolism in the thalamus and frontal, parietal, and posterior cingulate regions in $\mathrm{MCI}^{38}$. Reduced glucose metabolism in anterior and posterior cingulate cortices, retrosplenial, precuneus, parietal cortex, hippocampus and thalamus was also observed in cognitively healthy middle-aged $A P O E$ - $\varepsilon 4$ carriers $^{39}$, suggesting that metabolic tissue changes in regions beyond the hippocampus can already occur at asymptomatic stages ${ }^{40}$.

While PET imaging is sensitive to metabolic changes and can identify amyloid- $\beta$ and tau burden ${ }^{41}$, it is invasive and expensive and, therefore, difficult to scale up. Recent advances in non-invasive multi-parametric quantitative MRI (qMRI) methods can reveal subtle microstructural brain changes and promise to provide alternative imaging markers that may be sensitive to early risk-related changes. Up to now qMRI measurements have primarily been studied in LOAD patients and animal models, thus evidence with regards to the effects of risk factors in asymptomatic individuals is sparse.

To address this gap in the literature, we went beyond morphological analyses by employing multi-parametric qMRI to study the effects of $A P O E-\varepsilon 4$, Family History (FH) of dementia and obesity on cortical and subcortical grey matter in 165 asymptomatic individuals from the Cardiff Ageing and Risk of Dementia Study (CARDS) ${ }^{42-44}$ (Table 1). More specifically we applied indices sensitive to neurite dispersion and density, free water, myelin and cell metabolism from Neurite Orientation Density and Dispersion Imaging (NODDI ${ }^{45}$, quantitative magnetization transfer (qMT) $)^{46-49}$ and $\mathrm{T}_{1}$-relaxometry ${ }^{50}$ (Table 2).

NODDI fits a three-compartment biophysical tissue model to diffusion-weighted data acquired with a twoshell (b-values of $1200 \mathrm{~s} / \mathrm{mm}^{2}$ and $2400 \mathrm{~s} / \mathrm{mm}^{2}$ ) High Angular Resolution Diffusion Imaging (HARDI) ${ }^{51}$ protocol to separate isotropic from intra- and extracellular diffusion compartments ${ }^{45}$. This allows the calculation of the isotropic signal fraction (ISOSF), an estimate of free water, and the intracellular signal fraction (ICSF), i.e. the fraction of the tissue comprised of neurites. In addition, NODDI yields the orientation dispersion index (ODI) that reflects the spatial configuration of neurite structures (Table 2). Recent studies reported ICSF and ODI reductions in grey and white matter of patients with $\mathrm{MCI}, \mathrm{LOAD}$ and young onset $\mathrm{AD}^{52-54}$. For instance, Fu et al. (2019) found decreased ICSF and ODI in the corpus callosum in MCI and LOAD patients, while Colgan et al. ${ }^{55}$ reported positive correlations between ICSF and histological measurements of hyperphosphorylated tau protein in the hippocampus of $\mathrm{rTg} 4510$ mice.

The qMT method models the exchange rate between macromolecular protons and protons in surrounding free water when macromolecular protons are selectively saturated by a radiofrequency pulse with a frequency that is off-resonance for protons in free water ${ }^{46-49}$. This allows the quantification of a number of parameters including the macromolecular proton fraction (MPF) and the magnetization transfer exchange rate $k_{f}{ }^{49}$. In combined neuroimaging and histology studies of Shiverer mice and puppies ${ }^{56-58}$, MPF has been shown to be highly sensitive to the myelin content in white matter such that MPF increases with the amount of myelin. MPF in the anterior hippocampus was also found to distinguish healthy controls from MCI and LOAD patients ${ }^{59}$. Furthermore, MCI 


\begin{tabular}{|c|c|c|c|}
\hline MRI modality & Index & Apparent grey matter property & Hypothesised changes with LOAD risk \\
\hline \multirow{3}{*}{ Diffusion NODDI } & ICSF & Neurite density & Increases with tau pathology $y^{55} /$ Reduction in $\mathrm{MCI}$ and $\mathrm{AD}$ patients $\mathrm{s}^{52-54}$ \\
\hline & ODI & Neurite dispersion & Increase/Reduction \\
\hline & ISOSF & Free water & Increase \\
\hline \multirow[b]{2}{*}{ qMT } & MPF & Macromolecules (e.g. myelin) & Reduction \\
\hline & $k_{f}$ & Mitochondrial metabolism & $\begin{array}{l}\text { Increase in acute inflammation }{ }^{83} ; \\
\text { Reduction in low-level inflammation }{ }^{125} \text { and in MCI and AD patients }\end{array}$ \\
\hline Relaxometry & $\mathrm{R}_{1}$ & free water, myelin, iron & Increase/Reduction ${ }^{62}$ \\
\hline
\end{tabular}

Table 2. Overview of the quantitative microstructural indices and their interpretation in grey matter. $A D$ Alzheimer's disease, ICSF intracellular signal fraction, ISOSF isotropic signal fraction, $k_{f}$ forward exchange rate, $M C I$ mild cognitive impairment, $M P F$ macromolecular proton fraction, NODDI neurite orientation dispersion and density imaging, $O D I$ orientation dispersion index, $q M T$ quantitative magnetization transfer.

and LOAD patients exhibit a reduced rate of magnetization transfer $k_{f}$ in grey and white matter ${ }^{59-61}$ suggesting reduced cell metabolism ${ }^{60}$. Finally, indices from relaxometry imaging such as the longitudinal relaxation rate $\mathrm{R}_{1}$ have been proposed as non-invasive biomarkers of LOAD ${ }^{62} . \mathrm{R}_{1}$ values are influenced by microstructural characteristics such as tissue density, macromolecular, protein and lipid composition, and paramagnetic atoms. A number of patient and preclinical studies have reported increases in $\mathrm{R}_{1}$ that may reflect LOAD pathology, although the precise mechanisms underpinning these changes remain unknown (see for review ${ }^{62}$ ).

Here, we characterised age and risk-related differences in mean values of ICSF, ISOSF, ODI, MPF, $k_{f}$ and $\mathrm{R}_{1}$ across cortical and subcortical grey matter regions that were segmented from $\mathrm{T}_{1}$-weighted images with the FreeSurfer image analysis suite (version 5.3) ${ }^{63}$. Microstructural changes were compared with differences in standard morphological metrics of cortical thickness and subcortical volumes. We expected to see risk effects in brain regions known to be early affected in LOAD including limbic regions of the hippocampus, parahippocampus, entorhinal cortex, posterior cingulate cortex as well as thalamus ${ }^{2,4,34,64}$. We hypothesised that APOE-ع4, a positive $\mathrm{FH}$, and central obesity [measured with the Waist-Hip-Ratio (WHR)] would be associated with reduced ICSF, $\mathrm{R}_{1}, \mathrm{MPF}$ and $k_{f}$ as well as with increased ISOSF and ODI but with no differences in cortical thickness and/or subcortical volume. In addition, we expected to see the largest differences in those individuals at greatest risk, i.e. in obese $A P O E-\varepsilon 4$ carriers with a positive $\mathrm{FH}$.

\section{Results}

Microstructural and morphological dependent variables were fitted to a general linear model in SPSS version $26^{65}$. All data were examined for outliers defined as above or below three times of the interquartile range (75th percentile value-25th percentile value). This led to an exclusion of $0.6 \%$ of the microstructural but no exclusions of the morphological data.

Separate multivariate analyses of covariance (MANCOVA) were carried out to test for the effects of $A P O E$ genotype $(\varepsilon 4+, \varepsilon 4-), \mathrm{FH}(\mathrm{FH}+, \mathrm{FH}-)$ and WHR (WHR +, WHR-) on brain morphology (cortical thickness and subcortical volume measures) and on each of the microstructural indices (MPF, $k_{f}, \mathrm{R}_{1}$, ISOSF, ICSF, ODI) across 68 cortical and 14 subcortical regions of interest, whilst controlling for age, sex, and IQ estimates from the revised National Adult Reading Test (NART-R) ${ }^{66}$. Significant omnibus effects were further investigated with post-hoc comparisons across all outcome measures. All first and post-hoc models were corrected for multiple comparisons with a False Discovery Rate (FDR) of $5 \%$ using the Benjamini-Hochberg procedure ${ }^{67}$ ( $\mathrm{p}_{\mathrm{BHadj}}$ ). As the aim of the study was to explore microstructural indices that could potentially provide novel biomarkers of dementia risk in future studies, a false positive rate of below $5 \%$ was regarded as an acceptable threshold to control for false positives while minimising the risk of missing any true risk-related microstructural differences. Information about effects sizes was provided with the partial eta squared index $\eta_{\mathrm{p}}{ }^{2}$ for MANCOVA analyses, Cohen's $d_{z}$ for group comparisons and Pearson's $r$ for correlational analyses.

MANCOVAs of microstructural qMT metrics. MPF omnibus effects. There were main effects of $\operatorname{sex}\left[\mathrm{F}(78,46)=2.2, \mathrm{p}_{\mathrm{BHadj}}=0.015, \eta_{\mathrm{p}}{ }^{2}=0.8\right]$ and of $A P O E$ genotype $\left[\mathrm{F}(78,46)=2.6, \mathrm{p}_{\mathrm{BHadj}}<0.001, \eta_{\mathrm{p}}{ }^{2}=0.8\right]$ but not of FH ( $\left.\mathrm{p}_{\mathrm{BHadj}}=0.137\right)$, WHR ( $\left.\mathrm{p}_{\mathrm{BHadj}}=0.348\right)$, age $\left(\mathrm{p}_{\mathrm{BHadj}}=0.385\right)$ or NART-IQ $\left(\mathrm{p}_{\mathrm{BHadj}}=0.497\right)$. There were no interaction effects between $A P O E$ and $\mathrm{FH}\left(\mathrm{p}_{\mathrm{BHadj}}=1.000\right), A P O E$ and WHR ( $\left.\mathrm{p}_{\mathrm{BHadj}}=0.974\right), \mathrm{FH}$ and WHR $\left(\mathrm{p}_{\mathrm{BHadj}}=1.000\right)$ or $A P O E, \mathrm{FH}$ and WHR $\left(\mathrm{p}_{\mathrm{BHadj}}=0.935\right)$. 
MPF post-hoc effects. APOE- 44 carriers relative to non-carriers had lower MPF in the left thalamus (Table 3) (Fig. 1). Women had higher MPF than men in the left and right rostral middle frontal cortices, in the left superior temporal cortex and the right transverse temporal cortex (Table 3) (Fig. 2).

$R_{1}$ omnibus effects. A significant omnibus effect was only observed for $A P O E$ genotype $[\mathrm{F}(82,43)=2.1$, $\left.\mathrm{p}_{\mathrm{BHadj}}=0.040, \eta_{\mathrm{p}}{ }^{2}=0.08\right]$. No main effects were present for $\mathrm{FH}\left(\mathrm{p}_{\mathrm{BHadj}}=0.215\right)$, WHR $\left(\mathrm{p}_{\mathrm{BHadj}}=0.167\right)$, age $\left(\mathrm{p}_{\mathrm{BHadj}}=0.085\right)$ sex $\left(\mathrm{p}_{\mathrm{BHadj}}=0.060\right)$ or NART-IQ $\left(\mathrm{p}_{\mathrm{BHadj}}=0.866\right)$ and no interaction effects between $A P O E$ and FH $\left(\mathrm{p}_{\mathrm{BHadj}}=0.256\right), A P O E$ and WHR ( $\left.\mathrm{p}_{\text {BHadj }}=0.582\right), \mathrm{FH}$ and WHR ( $\left.\mathrm{p}_{\text {BHadj }}=0.782\right)$ or $A P O E, \mathrm{FH}$ and WHR $\left(\mathrm{p}_{\mathrm{BHadj}}=0.548\right)$ were observed.

$R_{1}$ post-hoc effects. No APOE post-hoc effects survived FDR correction (see Supplementary Table 1).

$k_{f}$ omnibus effects. There were no significant main effects of $A P O E\left(\mathrm{p}_{\mathrm{BHadj}}=0.813\right), \mathrm{FH}\left(\mathrm{p}_{\mathrm{BHadj}}=0.908\right), \mathrm{WHR}$ $\left(\mathrm{p}_{\mathrm{BHadj}}=1.000\right)$, age $\left(\mathrm{p}_{\mathrm{BHadj}}=0.075\right)$, sex $\left(\mathrm{p}_{\mathrm{BHadj}}=0.975\right)$ or NART-IQ $\left(\mathrm{p}_{\mathrm{BHadj}}=0.870\right)$ and no interaction effects between $A P O E$ and FH ( $\left.\mathrm{p}_{\text {BHadj }}=0.888\right), A P O E$ and WHR ( $\left.\mathrm{p}_{\text {BHadj }}=0.840\right), \mathrm{FH}$ and WHR ( $\left.\mathrm{p}_{\text {BHadj }}=0.090\right)$ or APOE, $\mathrm{FH}$ and WHR ( $\left.\mathrm{p}_{\mathrm{BHadj}}=0.436\right)$.

MANCOVAs of microstructural NODDI metrics. ISOSF omnibus effects. There were main effects for age $\left[\mathrm{F}(78,42)=2.0, \mathrm{p}_{\mathrm{BHadj}}=0.03, \eta_{\mathrm{p}}{ }^{2}=0.8\right]$, sex $\left[\mathrm{F}(78,42)=3.4, \mathrm{p}_{\mathrm{BHadj}}<0.001, \eta_{\mathrm{p}}{ }^{2}=0.9\right]$, and NART-IQ $\left[\mathrm{F}(78,42)=2.2, \mathrm{p}_{\mathrm{BHadj}}=0.020, \eta_{\mathrm{p}}{ }^{2}=0.8\right]$. No main effects were present for the risk factors of $A P O E\left(\mathrm{p}_{\mathrm{BHadj}}=1.000\right)$, $\mathrm{FH}\left(\mathrm{p}_{\mathrm{BHadj}}=0.060\right)$ or WHR $\left(\mathrm{p}_{\mathrm{BHadj}}=0.717\right)$ and no interaction effects between $A P O E$ and $\mathrm{FH}\left(\mathrm{p}_{\mathrm{BHadj}}=0.374\right)$, $A P O E$ and WHR ( $\left.\mathrm{p}_{\text {BHadj }}=0.551\right), \mathrm{FH}$ and WHR ( $\left.\mathrm{p}_{\mathrm{BHadj}}=0.986\right)$ or $A P O E, \mathrm{FH}$ and WHR ( $\left.\mathrm{p}_{\mathrm{BHadj}}=0.678\right)$ were observed.

ISOSF post-hoc effects. Ageing was associated with bilateral increases in ISOSF in medial regions including the cingulate, precuneus and cuneus cortices and in lateral regions including superior temporal, supramarginal, postcentral, pars opercularis and insula cortices. Age-related increases in ISOSF were also observed in left middle temporal and pars triangularis regions as well as in subcortical hippocampi, thalami, nuclei accumbens and right putamen (Table 4) (Fig. 3). Men relative to women had higher ISOSF in widespread frontal, temporal, parietal and cingulate cortices and in caudate nuclei, hippocampi, thalami and right nucleus accumbens (Table 4) (Fig. 2). In addition, NART-IQ correlated positively with ISOSF in the superior temporal sulci (left: $\mathrm{r}=0.253$, $\mathrm{p}_{\mathrm{BHadj}}=0.008$; right: $\left.\mathrm{r}=0.241, \mathrm{p}_{\mathrm{BHadj}}=0.006\right)$, left superior parietal $\left(\mathrm{r}=0.227, \mathrm{p}_{\mathrm{BHadj}}=0.006\right)$, and right lingual $\left(\mathrm{r}=0.182, \mathrm{p}_{\mathrm{BHadj}}=0.026\right)$ cortices (Table 4$)$. After partialling out of age only correlations on the left hemisphere remained significant [superior parietal cortex $\left[\left(\mathrm{r}=0.206, \mathrm{p}_{\mathrm{BHadj}}=0.048\right)\right.$, superior temporal sulcus $(\mathrm{r}=0.197$, $\left.\left.\mathrm{p}_{\mathrm{BHadj}}=0.032\right)\right]$ but those on the right did not [superior temporal sulcus $\left(\mathrm{p}_{\mathrm{BHadj}}=0.053\right)$, lingual $\left.\left(\mathrm{p}_{\mathrm{BHadj}}=0.08\right)\right]$.

ODI omnibus effects. There was a significant main effect of age $\left[\mathrm{F}(78,51)=2.0, \mathrm{p}_{\mathrm{BHadj}}=0.040, \mathrm{\eta}_{\mathrm{p}}{ }^{2}=0.8\right]$ and a significant interaction effect between $\mathrm{FH}$ and $\operatorname{WHR}\left[\mathrm{F}(78,51)=2.3, \mathrm{p}_{\mathrm{BHadj}}=0.010, \eta_{\mathrm{p}}{ }^{2}=0.8\right]$ but no main effects for $\operatorname{sex}\left(\mathrm{p}_{\text {BHadj }}=0.270\right)$, NART-IQ $\left(\mathrm{p}_{\text {BHadj }}=0.497\right), A P O E\left(\mathrm{p}_{\text {BHadj }}=0.153\right), \mathrm{FH}\left(\mathrm{p}_{\text {BHadj }}=0.520\right)$ or WHR $\left(\mathrm{p}_{\text {BHadj }}=0.330\right)$ and no interaction effects between $A P O E$ and $\mathrm{FH}$ ( $\left.\mathrm{p}_{\mathrm{BHadj}}=0.436\right), A P O E$ and WHR ( $\left.\mathrm{p}_{\mathrm{BHadj}}=0.295\right)$ or $A P O E, \mathrm{FH}$ and WHR ( $\mathrm{p}_{\text {BHadj }}=0.228$ ) were observed.

ODI post-hoc effects. Age-related increases in ODI were observed in left hippocampus, amygdala, caudate and right transverse temporal cortex (Table 5) (Fig. 3).

Post-hoc effects for the interaction between FH and WHR did not survive 5\% FDR correction (Supplementary Table 2).

ICSF effects. There were no significant main or interaction effects on ICSF [age $\left(\mathrm{p}_{\text {BHadj }}=0.170\right)$, sex $\left(\mathrm{p}_{\text {BHadj }}=0.130\right)$, NART-IQ $\left(\mathrm{p}_{\text {BHadj }}=0.451\right), A P O E\left(\mathrm{p}_{\text {BHadj }}=0.324\right), \mathrm{FH}\left(\mathrm{p}_{\text {BHadj }}=0.342\right)$, WHR $\left(\mathrm{p}_{\text {BHadj }}=0.517\right)$, $A P O E \times \mathrm{FH}\left(\mathrm{p}_{\text {BHadj }}=0.541\right), A P O E \times \mathrm{WHR}\left(\mathrm{p}_{\text {BHadj }}=0.236\right), \mathrm{FH} \times \mathrm{WHR}\left(\mathrm{p}_{\text {BHadj }}=0.883\right), A P O E \times \mathrm{FH} \times \mathrm{WHR}$ $\left.\left(\mathrm{p}_{\text {BHadj }}=0.912\right)\right]$.

MANCOVA on cortical thickness and subcortical volume (ICV corrected). Omnibus effects. There were main effects for age $\left[\mathrm{F}(82,68)=1.8, \mathrm{p}_{\mathrm{BHadj}}=0.035, \eta_{\mathrm{p}}{ }^{2}=0.7\right]$ and $\operatorname{sex}\left[\mathrm{F}(82,68)=1.9, \mathrm{p}_{\mathrm{BHadj}}=0.040, \eta_{\mathrm{p}}{ }^{2}=0.7\right]$. No main effects were observed for APOE $\left(\mathrm{p}_{\mathrm{BHadj}}=0.597\right)$, FH $\left(\mathrm{p}_{\mathrm{BHadj}}=0.144\right)$, WHR $\left(\mathrm{p}_{\mathrm{BHadj}}=0.152\right)$ or NART-IQ $\left(\mathrm{p}_{\mathrm{BHadj}}=0.651\right)$. No interaction effects between $A P O E$ and $\mathrm{FH}\left(\mathrm{p}_{\mathrm{BHadj}}=0.844\right), A P O E$ and $\mathrm{WHR}\left(\mathrm{p}_{\mathrm{BHadj}}=0.978\right)$, FH and WHR $\left(\mathrm{p}_{\mathrm{BHadj}}=0.053\right)$ or $A P O E, \mathrm{FH}$ and WHR $\left(\mathrm{p}_{\mathrm{BHadj}}=0.123\right)$ were observed.

Post-hoc effects. Ageing was associated with widespread thinning in bilateral frontal, temporal, and parietal cortical regions as well as with volume loss in subcortical structures, i.e. in the left hippocampus, left nucleus accumbens, bilateral thalami and putamen (Table 6) (Fig. 3). Women relative to men had larger volumes in left hippocampus, left nucleus accumbens, left putamen, right caudate and right pallidum. They also had larger cortical thickness in the right isthmus cingulate but lower cortical thickness in the left insula (Table 6) (Fig. 2).

Exploring interaction effects between APOE, age and sex. Potential interaction effects between $A P O E$, age and sex on left thalamus MPF were explored. Univariate analysis of variance revealed an effect of 


\begin{tabular}{|c|c|c|c|c|}
\hline Effect & Side & ROI & $F_{(1,123)}$-value & $\mathbf{p}_{\text {BHadj }}$ \\
\hline \multirow{56}{*}{ APOE } & \multirow{7}{*}{ Left } & Accumbens & 3.985 & 0.214 \\
\hline & & Amygdala & 0.171 & 0.869 \\
\hline & & Caudate & 6.710 & 0.090 \\
\hline & & Hippocampus & 5.327 & 0.143 \\
\hline & & Pallidum & 0.099 & 0.891 \\
\hline & & Putamen & 1.416 & 0.511 \\
\hline & & Thalamus & 10.772 & 0.026 \\
\hline & \multirow{7}{*}{ Right } & Accumbens & 0.310 & 0.790 \\
\hline & & Amygdala & 0.125 & 0.868 \\
\hline & & Caudate & 3.433 & 0.264 \\
\hline & & Hippocampus & 6.700 & 0.095 \\
\hline & & Pallidum & 0.039 & 0.919 \\
\hline & & Putamen & 1.226 & 0.561 \\
\hline & & Thalamus & 5.233 & 0.144 \\
\hline & \multirow{31}{*}{ Left } & Banks of superior temporal sulcus & 3.424 & 0.261 \\
\hline & & Caudal anterior cingulate & 1.518 & 0.483 \\
\hline & & Cuneus & 0.631 & 0.689 \\
\hline & & Entorhinal & 0.002 & 0.986 \\
\hline & & Frontal pole & 2.579 & 0.320 \\
\hline & & Fusiform & 0.771 & 0.669 \\
\hline & & Inferior parietal & 0.886 & 0.631 \\
\hline & & Inferior temporal & 0.942 & 0.635 \\
\hline & & Insula & 6.754 & 0.097 \\
\hline & & Lateral occipital & 0.307 & 0.788 \\
\hline & & Lateral orbito frontal & 0.355 & 0.777 \\
\hline & & Lingual & 0.641 & 0.690 \\
\hline & & Medial orbito frontal & 0.001 & 0.993 \\
\hline & & Middle temporal & 2.653 & 0.318 \\
\hline & & Paracentral & 0.035 & 0.924 \\
\hline & & Parahippocampal & 0.150 & 0.865 \\
\hline & & Pars opercularis & 8.341 & 0.097 \\
\hline & & Pars orbitalis & 0.028 & 0.932 \\
\hline & & Pars triangularis & 0.019 & 0.945 \\
\hline & & Postcentral & 2.459 & 0.331 \\
\hline & & Posterior cingulate & 1.065 & 0.592 \\
\hline & & Precentral & 3.040 & 0.297 \\
\hline & & Precuneus & 0.000 & 0.997 \\
\hline & & Rostral anterior cingulate & 0.531 & 0.714 \\
\hline & & Rostral middle frontal & 0.112 & 0.880 \\
\hline & & Superior frontal & 0.515 & 0.719 \\
\hline & & Superior parietal & 0.222 & 0.836 \\
\hline & & Superior temporal & 1.096 & 0.594 \\
\hline & & Supramarginal & 2.657 & 0.312 \\
\hline & & Temporal pole & 3.597 & 0.252 \\
\hline & & Transverse temporal & 5.752 & 0.117 \\
\hline & \multirow{11}{*}{ Right } & Banks of superior temporal sulcus & 0.085 & 0.892 \\
\hline & & Caudal anterior cingulate & 6.693 & 0.100 \\
\hline & & Cuneus & 0.077 & 0.897 \\
\hline & & Entorhinal & 0.088 & 0.892 \\
\hline & & Frontal pole & 0.070 & 0.882 \\
\hline & & Fusiform & 2.047 & 0.416 \\
\hline & & Inferior parietal & 0.736 & 0.673 \\
\hline & & Inferior temporal & 0.162 & 0.865 \\
\hline & & Insula & 4.235 & 0.198 \\
\hline & & Isthmus cingulate & 0.927 & 0.635 \\
\hline & & Lateral occipital & 0.072 & 0.891 \\
\hline
\end{tabular}




\begin{tabular}{|c|c|c|c|c|}
\hline Effect & Side & ROI & $F_{(1,123)}$-value & $\mathbf{p}_{\text {BHadj }}$ \\
\hline & & Lateral orbito frontal & 0.785 & 0.668 \\
\hline & & Lingual & 3.499 & 0.262 \\
\hline & & Medial orbito frontal & 1.979 & 0.407 \\
\hline & & Middle temporal & 0.130 & 0.876 \\
\hline & & Paracentral & 0.071 & 0.887 \\
\hline & & Parahippocampal & 1.994 & 0.409 \\
\hline & & Pars opercularis & 1.551 & 0.493 \\
\hline & & Pars orbitalis & 0.511 & 0.714 \\
\hline & & Pars triangularis & 0.001 & 0.986 \\
\hline & & Pericalcerine & 0.875 & 0.629 \\
\hline & & Postcentral & 0.074 & 0.895 \\
\hline & & Posterior cingulate & 1.341 & 0.532 \\
\hline & & Precentral & 0.303 & 0.784 \\
\hline & & Precuneus & 0.198 & 0.854 \\
\hline & & Rostral anterior cingulate & 1.850 & 0.429 \\
\hline & & Rostral middle frontal & 0.151 & 0.858 \\
\hline & & Superior frontal & 0.026 & 0.932 \\
\hline & & Superior parietal & 1.548 & 0.488 \\
\hline & & Superior temporal & 1.148 & 0.579 \\
\hline & & Supramarginal & 0.167 & 0.866 \\
\hline & & Temporal pole & 0.764 & 0.665 \\
\hline & & Transverse temporal & 0.155 & 0.867 \\
\hline \multirow{34}{*}{ Sex } & \multirow{7}{*}{ Left } & Accumbens & 0.353 & 0.784 \\
\hline & & Amygdala & 0.014 & 0.956 \\
\hline & & Caudate & 1.918 & 0.418 \\
\hline & & Hippocampus & 0.684 & 0.673 \\
\hline & & Pallidum & 1.079 & 0.594 \\
\hline & & Putamen & 2.12 & 0.405 \\
\hline & & Thalamus & 2.668 & 0.321 \\
\hline & \multirow{7}{*}{ Right } & Accumbens & 0.126 & 0.874 \\
\hline & & Amygdala & 0.000 & 0.993 \\
\hline & & Caudate & 0.046 & 0.912 \\
\hline & & Hippocampus & 0.223 & 0.842 \\
\hline & & Pallidum & 0.697 & 0.673 \\
\hline & & Putamen & 2.678 & 0.324 \\
\hline & & Thalamus & 0.571 & 0.710 \\
\hline & \multirow{20}{*}{ Left } & Banks of superior temporal sulcus & 0.559 & 0.711 \\
\hline & & Caudal anterior cingulate & 0.459 & 0.742 \\
\hline & & Cuneus & 7.712 & 0.093 \\
\hline & & Entorhinal & 5.902 & 0.115 \\
\hline & & Frontal pole & 4.243 & 0.204 \\
\hline & & Fusiform & 0.007 & 0.971 \\
\hline & & Inferior parietal & 6.242 & 0.104 \\
\hline & & Inferior temporal & 0.191 & 0.854 \\
\hline & & Insula & 1.298 & 0.541 \\
\hline & & Lateral occipital & 0.063 & 0.888 \\
\hline & & Lateral orbito frontal & 0.002 & 0.992 \\
\hline & & Lingual & 3.095 & 0.293 \\
\hline & & Medial orbito frontal & 2.921 & 0.298 \\
\hline & & Middle temporal & 2.496 & 0.331 \\
\hline & & Paracentral & 0.009 & 0.968 \\
\hline & & Parahippocampal & 7.180 & 0.104 \\
\hline & & Pars opercularis & 1.169 & 0.578 \\
\hline & & Pars orbitalis & 1.524 & 0.488 \\
\hline & & Pars triangularis & 7.929 & 0.085 \\
\hline & & Postcentral & 0.903 & 0.638 \\
\hline
\end{tabular}




\begin{tabular}{|c|c|c|c|c|}
\hline Effect & Side & ROI & $F_{(1,123)}$-value & $\mathbf{p}_{\text {BHadj }}$ \\
\hline & & Posterior cingulate & 15.379 & $<0.001$ \\
\hline & & Precentral & 0.726 & 0.664 \\
\hline & & Precuneus & 4.327 & 0.201 \\
\hline & & Rostral anterior cingulate & 0.727 & 0.669 \\
\hline & & Rostral middle frontal & 18.725 & $<0.001$ \\
\hline & & Superior frontal & 4.349 & 0.202 \\
\hline & & Superior parietal & 1.629 & 0.474 \\
\hline & & Superior temporal & 13.584 & $<0.001$ \\
\hline & & Supramarginal & 7.837 & 0.104 \\
\hline & & Temporal pole & 3.766 & 0.238 \\
\hline & & Transverse temporal & 7.374 & 0.096 \\
\hline & \multirow{33}{*}{ Right } & BANKS of superior temporal sulcus & 2.881 & 0.292 \\
\hline & & Caudal anterior cingulate & 4.038 & 0.215 \\
\hline & & Cuneus & 7.177 & 0.089 \\
\hline & & Entorhinal & 2.004 & 0.413 \\
\hline & & Frontal pole & 4.610 & 0.196 \\
\hline & & Fusiform & 0.097 & 0.886 \\
\hline & & Inferior parietal & 1.757 & 0.442 \\
\hline & & Inferior temporal & 0.352 & 0.771 \\
\hline & & Insula & 2.943 & 0.308 \\
\hline & & Isthmus cingulate & 0.443 & 0.746 \\
\hline & & Lateral occipital & 0.297 & 0.782 \\
\hline & & Lateral orbito frontal & 0.356 & 0.790 \\
\hline & & Lingual & 3.196 & 0.289 \\
\hline & & Medial orbito frontal & 4.570 & 0.195 \\
\hline & & Middle temporal & 0.360 & 0.793 \\
\hline & & Paracentral & 0.425 & 0.752 \\
\hline & & Parahippocampal & 0.975 & 0.625 \\
\hline & & Pars opercularis & 0.340 & 0.774 \\
\hline & & Pars orbitalis & 0.892 & 0.636 \\
\hline & & Pars triangularis & 6.046 & 0.106 \\
\hline & & Pericalcerine & 0.553 & 0.708 \\
\hline & & Postcentral & 2.934 & 0.301 \\
\hline & & Posterior cingulate & 1.783 & 0.441 \\
\hline & & Precentral & 2.025 & 0.415 \\
\hline & & Precuneus & 0.597 & 0.702 \\
\hline & & Rostral anterior cingulate & 3.205 & 0.282 \\
\hline & & Rostral middle frontal & 11.339 & 0.031 \\
\hline & & Superior frontal & 8.639 & 0.089 \\
\hline & & Superior parietal & 4.557 & 0.188 \\
\hline & & Superior temporal & 7.319 & 0.083 \\
\hline & & Supramarginal & 2.903 & 0.295 \\
\hline & & Temporal pole & 6.534 & 0.093 \\
\hline & & Transverse temporal & 14.344 & $<0.001$ \\
\hline
\end{tabular}

Table 3. Post-hoc effects of $A P O E$ genotype and sex on the macromolecular proton fraction (MPF). $\mathrm{p}_{\mathrm{BHadj}}$, $5 \%$ False Discovery Rate Benjamini-Hochberg adjusted $p$ value; $R O I$ region of interest. Significant results are highlighted in bold.

$A P O E[\mathrm{~F}(1,141)=5.7, p=0.018]$ and age $[\mathrm{F}(2,141)=3.7, p=0.027]$ but no interaction effects between $A P O E$ and age $(p=0.700)$ or $A P O E$ and sex $(p=0.900)$.

Exploring moderator effects of blood pressure and markers of inflammation. We then explored with two separate analyses of covariances whether controlling for differences in (i) systolic and diastolic blood pressure (BP) and (ii) inflammation-related measures of C-Reactive Protein (CRP), Interleukin-8 (IL-8) and leptin/adiponectin ratio (LAR) would account for the effect of $A P O E$ on left thalamus MPF. 


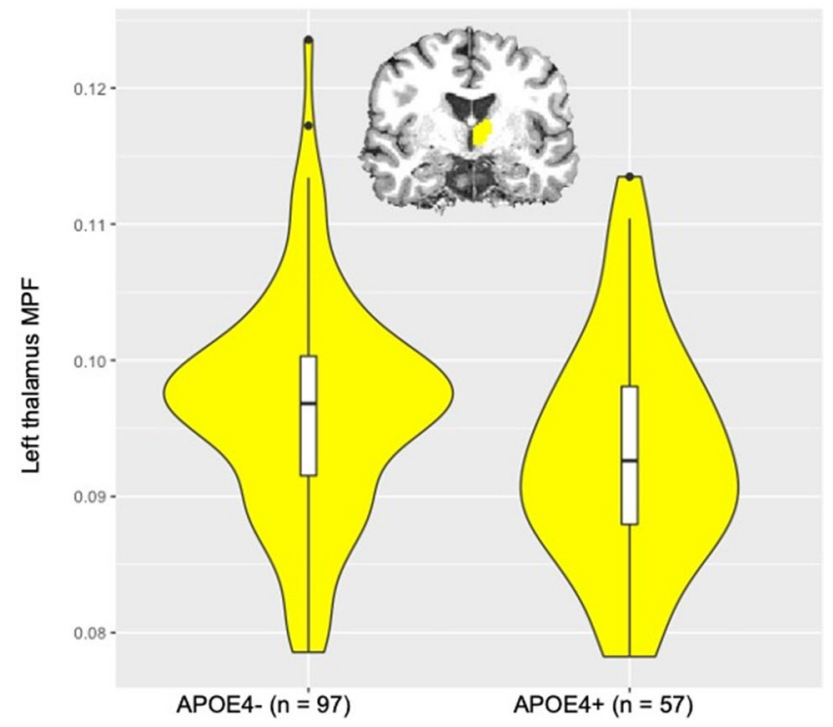

Figure 1. Violin plots with overlaid box plots of the difference in the macromolecular proton fraction (MPF) in the left thalamus between $A P O E$ - $\varepsilon 4$ carriers $(n=57)$ and non-carriers $(n=97)\left(p_{B H a d j}=0.026\right)$. Boxplots display the median and the interquartile range and violin plots the kernel probability density, i.e. the width of the yellow area represents the proportion of the data located there.

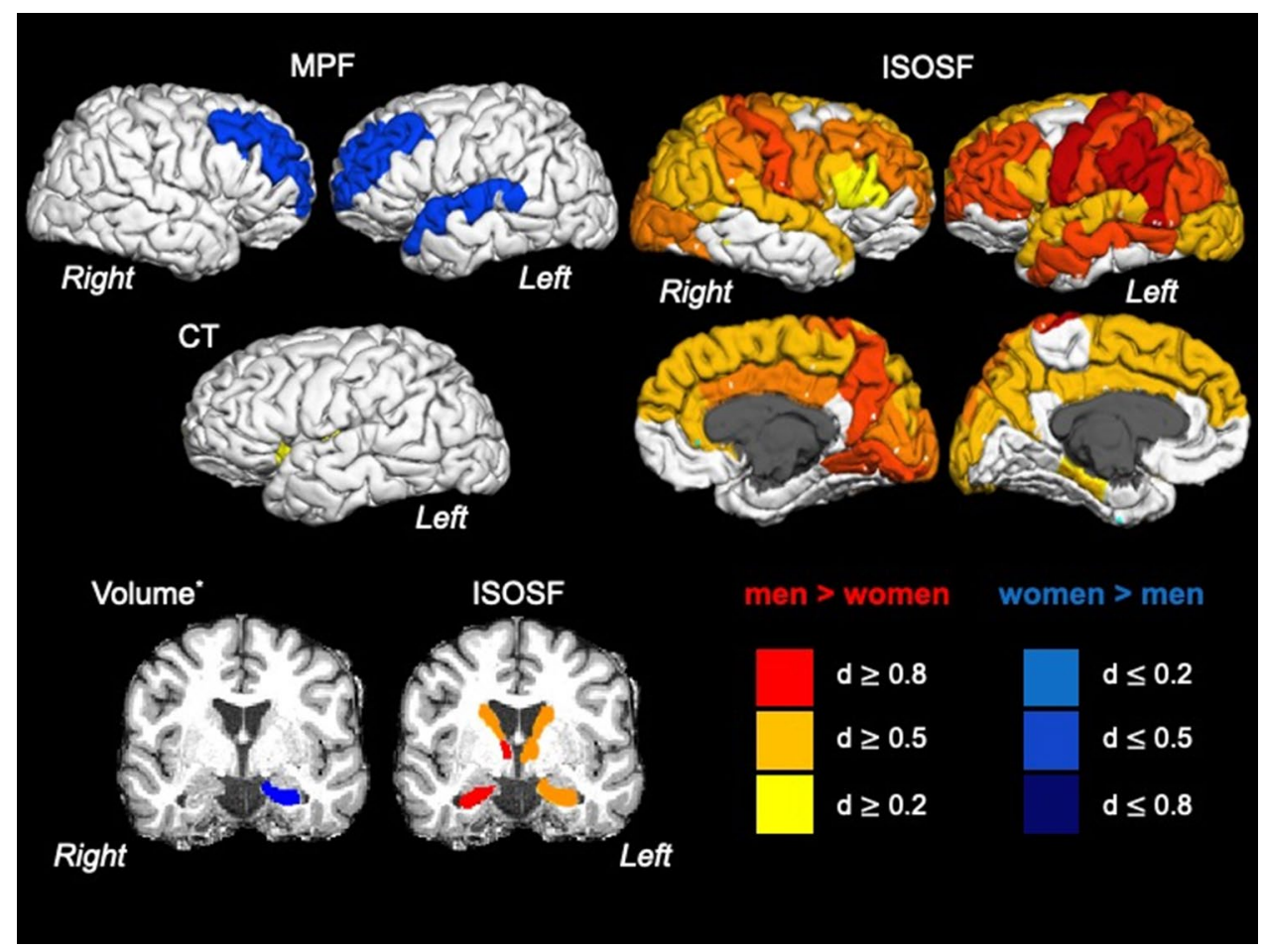

Figure 2. displays the effects of sex on cortical thickness (CT), subcortical volume (corrected for intracranial volume), isotropic signal fraction (ISOSF) and macromolecular proton fraction (MPF) across 34 cortical regions per hemisphere parcellated with the Desikan-Killiany atlas ${ }^{121}$ and seven subcortical regions per hemisphere (hippocampus, amygdala, thalamus, caudate, putamen, globus pallidus, nucleus accumbens). Region of interest segmentations were performed with FreeSurfer (version 5.3). Regions are colour-coded according to effect sizes indicated by Cohen's $\mathrm{d}^{126}$. Warm colours indicate positive and blue colours negative correlations. $\mathrm{L}=\mathrm{Left}$, $\mathrm{R}=$ Right. 


\begin{tabular}{|c|c|c|c|c|}
\hline Effect & Side & ROI & $F_{(1,119)}$-value & $\mathbf{p}_{\text {BHadj }}$ \\
\hline \multirow{56}{*}{ Age } & \multirow{7}{*}{ Left } & Accumbens & 16.946 & $<0.001$ \\
\hline & & Amygdala & 0.002 & 0.977 \\
\hline & & Caudate & 2.906 & 0.174 \\
\hline & & Hippocampus & 32.296 & $<0.001$ \\
\hline & & Pallidum & 0.741 & 0.544 \\
\hline & & Putamen & 3.705 & 0.121 \\
\hline & & Thalamus & 17.881 & $<0.001$ \\
\hline & \multirow{7}{*}{ Right } & Accumbens & 8.272 & 0.016 \\
\hline & & Amygdala & 0.090 & 0.847 \\
\hline & & Caudate & 4.359 & 0.090 \\
\hline & & Hippocampus & 20.305 & $<0.001$ \\
\hline & & Pallidum & 0.168 & 0.787 \\
\hline & & Putamen & 6.089 & 0.039 \\
\hline & & Thalamus & 21.716 & $<0.001$ \\
\hline & \multirow{31}{*}{ Left } & Banks of superior temporal sulcus & 12.121 & 0.003 \\
\hline & & Caudal anterior cingulate & 12.152 & 0.004 \\
\hline & & Cuneus & 17.203 & $<0.001$ \\
\hline & & Entorhinal & 0.170 & 0.788 \\
\hline & & Frontal pole & 0.667 & 0.559 \\
\hline & & Fusiform & 0.884 & 0.494 \\
\hline & & Inferior parietal & 6.381 & 0.035 \\
\hline & & Inferior temporal & 0.765 & 0.538 \\
\hline & & Insula & 17.457 & $<0.001$ \\
\hline & & Lateral occipital & 6.671 & 0.031 \\
\hline & & Lateral orbito frontal & 3.029 & 0.163 \\
\hline & & Lingual & 2.481 & 0.212 \\
\hline & & Medial orbito frontal & 6.335 & 0.035 \\
\hline & & Middle temporal & 11.334 & 0.004 \\
\hline & & Paracentral & 4.216 & 0.095 \\
\hline & & Parahippocampal & 0.125 & 0.819 \\
\hline & & Pars opercularis & 19.568 & $<0.001$ \\
\hline & & Pars orbitalis & 0.005 & 0.961 \\
\hline & & Pars triangularis & 15.445 & $<0.001$ \\
\hline & & Postcentral & 14.471 & $<0.001$ \\
\hline & & Posterior cingulate & 15.798 & $<0.001$ \\
\hline & & Precentral & 5.314 & 0.057 \\
\hline & & Precuneus & 19.354 & $<0.001$ \\
\hline & & Rostral anterior cingulate & 16.241 & $<0.001$ \\
\hline & & Rostral middle frontal & 5.017 & 0.067 \\
\hline & & Superior frontal & 1.173 & 0.410 \\
\hline & & Superior parietal & 0.963 & 0.470 \\
\hline & & Superior temporal & 25.891 & $<0.001$ \\
\hline & & Supramarginal & 16.621 & $<0.001$ \\
\hline & & Temporal pole & 1.219 & 0.410 \\
\hline & & Transverse temporal & 51.576 & $<0.001$ \\
\hline & \multirow{11}{*}{ Right } & Banks of superior temporal sulcus & 12.346 & 0.003 \\
\hline & & Caudal anterior cingulate & 7.267 & 0.025 \\
\hline & & Cuneus & 13.388 & $<0.001$ \\
\hline & & Entorhinal & 0.131 & 0.819 \\
\hline & & Frontal pole & 1.185 & 0.414 \\
\hline & & Fusiform & 0.108 & 0.835 \\
\hline & & Inferior parietal & 1.881 & 0.297 \\
\hline & & Inferior temporal & 1.475 & 0.366 \\
\hline & & Insula & 14.803 & $<0.001$ \\
\hline & & Isthmus cingulate & 6.659 & 0.031 \\
\hline & & Lateral occipital & 1.818 & 0.307 \\
\hline
\end{tabular}




\begin{tabular}{|c|c|c|c|c|}
\hline Effect & Side & ROI & $F_{(1,119)}$-value & $\mathbf{p}_{\text {BHadj }}$ \\
\hline & & Lateral orbito frontal & 1.286 & 0.406 \\
\hline & & Lingual & 7.195 & 0.024 \\
\hline & & Medial orbito frontal & 3.288 & 0.147 \\
\hline & & Middle temporal & 3.039 & 0.165 \\
\hline & & Paracentral & 0.702 & 0.556 \\
\hline & & PARAHIPPOCAMPAL & 1.158 & 0.412 \\
\hline & & Pars opercularis & 15.415 & $<0.001$ \\
\hline & & Pars orbitalis & 2.665 & 0.195 \\
\hline & & Pars triangularis & 0.523 & 0.605 \\
\hline & & Pericalcerine & 16.505 & $<0.001$ \\
\hline & & Postcentral & 6.318 & 0.034 \\
\hline & & Posterior cingulate & 18.89 & $<0.001$ \\
\hline & & Precentral & 4.015 & 0.104 \\
\hline & & Precuneus & 15.968 & $<0.001$ \\
\hline & & Rostral anterior cingulate & 12.476 & 0.003 \\
\hline & & Rostral middle frontal & 2.466 & 0.212 \\
\hline & & Superior frontal & 0.676 & 0.550 \\
\hline & & Superior parietal & 3.634 & 0.124 \\
\hline & & Superior temporal & 12.296 & 0.003 \\
\hline & & Supramarginal & 8.563 & 0.013 \\
\hline & & Temporal pole & 2.727 & 0.189 \\
\hline & & Transverse temporal & 44.346 & $<0.001$ \\
\hline \multirow{34}{*}{ Sex } & \multirow{7}{*}{ Left } & Accumbens & 4.687 & 0.078 \\
\hline & & Amygdala & 0.320 & 0.693 \\
\hline & & Caudate & 6.885 & 0.029 \\
\hline & & Hippocampus & 30.457 & $<0.001$ \\
\hline & & Pallidum & 3.735 & 0.120 \\
\hline & & Putamen & 0.886 & 0.497 \\
\hline & & Thalamus & 6.685 & 0.031 \\
\hline & \multirow{7}{*}{ Right } & Accumbens & 10.982 & 0.003 \\
\hline & & Amygdala & 3.110 & 0.161 \\
\hline & & Caudate & 8.610 & 0.013 \\
\hline & & Hippocampus & 37.739 & $<0.001$ \\
\hline & & Pallidum & 1.177 & 0.412 \\
\hline & & Putamen & 0.595 & 0.577 \\
\hline & & Thalamus & 28.188 & $<0.001$ \\
\hline & \multirow{20}{*}{ Left } & Banks of superior temporal sulcus & 9.745 & 0.007 \\
\hline & & Caudal anterior cingulate & 10.321 & 0.007 \\
\hline & & Cuneus & 14.189 & $<0.001$ \\
\hline & & Entorhinal & 2.097 & 0.263 \\
\hline & & Frontal pole & 1.317 & 0.400 \\
\hline & & Fusiform & 0.471 & 0.621 \\
\hline & & Inferior parietal & 19.193 & $<0.001$ \\
\hline & & Inferior temporal & 3.546 & 0.129 \\
\hline & & Insula & 14.093 & $<0.001$ \\
\hline & & Lateral occipital & 15.940 & $<0.001$ \\
\hline & & Lateral orbito frontal & 0.039 & 0.902 \\
\hline & & Lingual & 1.178 & 0.414 \\
\hline & & Medial orbito frontal & 3.411 & 0.138 \\
\hline & & Middle temporal & 17.995 & $<0.001$ \\
\hline & & Paracentral & 1.542 & 0.355 \\
\hline & & Parahippocampal & 14.537 & $<0.001$ \\
\hline & & Pars opercularis & 11.519 & 0.003 \\
\hline & & Pars orbitalis & 0.167 & 0.784 \\
\hline & & Pars triangularis & 16.204 & $<0.001$ \\
\hline & & Postcentral & 28.162 & $<0.001$ \\
\hline & & & & \\
\hline
\end{tabular}




\begin{tabular}{|c|c|c|c|c|}
\hline Effect & Side & ROI & $F_{(1,119)}$-value & $\mathbf{p}_{\text {BHadj }}$ \\
\hline & & Posterior cingulate & 16.237 & $<0.001$ \\
\hline & & Precentral & 22.987 & $<0.001$ \\
\hline & & Precuneus & 13.571 & $<0.001$ \\
\hline & & Rostral anterior cingulate & 4.385 & 0.088 \\
\hline & & Rostral middle frontal & 35.530 & $<0.001$ \\
\hline & & Superior frontal & 13.064 & $<0.001$ \\
\hline & & Superior parietal & 18.143 & $<0.001$ \\
\hline & & Superior temporal & 26.621 & $<0.001$ \\
\hline & & \begin{tabular}{|l|} 
Supramarginal \\
\end{tabular} & 42.479 & $<0.001$ \\
\hline & & Temporal pole & 4.436 & 0.088 \\
\hline & & Transverse temporal & 30.601 & $<0.001$ \\
\hline & & Banks of superior temporal sulcus & 14.697 & $<0.001$ \\
\hline & & Caudal anterior cingulate & 10.623 & 0.004 \\
\hline & & Cuneus & 24.330 & $<0.001$ \\
\hline & & Entorhinal & 0.491 & 0.616 \\
\hline & & Frontal pole & 0.684 & 0.557 \\
\hline & & Fusiform & 3.168 & 0.158 \\
\hline & & Inferior parietal & 6.885 & 0.030 \\
\hline & & Inferior temporal & 3.105 & 0.162 \\
\hline & & Insula & 4.265 & 0.094 \\
\hline & & Isthmus cingulate & 0.601 & 0.578 \\
\hline & & \begin{tabular}{|l|} 
Lateral occipital \\
\end{tabular} & 10.275 & 0.006 \\
\hline & & Lateral orbito frontal & 0.102 & 0.839 \\
\hline & & Lingual & 7.981 & 0.019 \\
\hline & & Medial orbito frontal & 3.038 & 0.166 \\
\hline & & Middle temporal & 5.352 & 0.055 \\
\hline & & Paracentral & 9.075 & 0.010 \\
\hline & Right & Parahippocampal & 3.733 & 0.121 \\
\hline & & Pars opercularis & 7.161 & 0.027 \\
\hline & & Pars orbitalis & 3.870 & 0.112 \\
\hline & & Pars triangularis & 5.958 & 0.042 \\
\hline & & Pericalcerine & 14.080 & $<0.001$ \\
\hline & & Postcentral & 19.109 & $<0.001$ \\
\hline & & Posterior cingulate & 14.954 & $<0.001$ \\
\hline & & Precentral & 17.777 & $<0.001$ \\
\hline & & Precuneus & 13.291 & $<0.001$ \\
\hline & & Rostral anterior cingulate & 5.785 & 0.046 \\
\hline & & Rostral middle frontal & 24.380 & $<0.001$ \\
\hline & & Superior frontal & 16.120 & $<0.001$ \\
\hline & & Superior parietal & 8.266 & 0.016 \\
\hline & & Superior temporal & 16.902 & $<0.001$ \\
\hline & & \begin{tabular}{|l|} 
Supramarginal \\
\end{tabular} & 16.983 & $<0.001$ \\
\hline & & \begin{tabular}{|l|} 
Temporal pole \\
\end{tabular} & 0.330 & 0.691 \\
\hline & & Transverse temporal & 37.792 & $<0.001$ \\
\hline \multirow{12}{*}{ NART-IQ } & \multirow{7}{*}{ Left } & Accumbens & 0.709 & 0.556 \\
\hline & & Amygdala & 3.741 & 0.120 \\
\hline & & Caudate & 0.016 & 0.932 \\
\hline & & Hippocampus & 0.065 & 0.864 \\
\hline & & Pallidum & 0.022 & 0.922 \\
\hline & & Putamen & 1.221 & 0.411 \\
\hline & & Thalamus & 0.000 & 0.995 \\
\hline & \multirow{5}{*}{ Right } & Accumbens & 0.022 & 0.924 \\
\hline & & Amygdala & 1.266 & 0.410 \\
\hline & & Caudate & 1.809 & 0.306 \\
\hline & & Hippocampus & 0.067 & 0.866 \\
\hline & & Pallidum & 0.206 & 0.764 \\
\hline
\end{tabular}




\begin{tabular}{|c|c|c|c|c|}
\hline Effect & Side & ROI & $F_{(1,119)}$-value & $\mathbf{p}_{\text {BHadj }}$ \\
\hline & & Putamen & 0.606 & 0.579 \\
\hline & & \begin{tabular}{|l|} 
Thalamus \\
\end{tabular} & 0.481 & 0.618 \\
\hline & \multirow{31}{*}{ Left } & Banks of superior temporal sulcus & 6.816 & 0.029 \\
\hline & & Caudal anterior cingulate & 0.035 & 0.901 \\
\hline & & Cuneus & 0.200 & 0.767 \\
\hline & & Entorhinal & 0.343 & 0.684 \\
\hline & & Frontal pole & 1.745 & 0.315 \\
\hline & & Fusiform & 0.039 & 0.904 \\
\hline & & Inferior parietal & 2.029 & 0.274 \\
\hline & & Inferior temporal & 0.019 & 0.925 \\
\hline & & Insula & 4.834 & 0.073 \\
\hline & & Lateral occipital & 0.306 & 0.697 \\
\hline & & Lateral orbito frontal & 0.037 & 0.901 \\
\hline & & Lingual & 0.621 & 0.574 \\
\hline & & Medial orbito frontal & 0.000 & 0.993 \\
\hline & & Middle temporal & 0.402 & 0.655 \\
\hline & & Paracentral & 0.199 & 0.764 \\
\hline & & Parahippocampal & 0.010 & 0.943 \\
\hline & & Pars opercularis & 0.207 & 0.768 \\
\hline & & Pars orbitalis & 1.006 & 0.459 \\
\hline & & Pars triangularis & 0.636 & 0.570 \\
\hline & & Postcentral & 1.370 & 0.388 \\
\hline & & Posterior cingulate & 1.243 & 0.411 \\
\hline & & Precentral & 0.401 & 0.653 \\
\hline & & Precuneus & 0.078 & 0.852 \\
\hline & & Rostral anterior cingulate & 0.582 & 0.581 \\
\hline & & Rostral middle frontal & 1.208 & 0.411 \\
\hline & & Superior frontal & 1.224 & 0.414 \\
\hline & & Superior parietal & 6.435 & 0.033 \\
\hline & & Superior temporal & 0.266 & 0.724 \\
\hline & & Supramarginal & 0.879 & 0.493 \\
\hline & & Temporal pole & 0.084 & 0.849 \\
\hline & & Transverse temporal & 2.832 & 0.180 \\
\hline & \multirow{23}{*}{ Right } & Banks of superior temporal sulcus & 6.815 & 0.030 \\
\hline & & Caudal anterior cingulate & 0.530 & 0.605 \\
\hline & & Cuneus & 2.829 & 0.179 \\
\hline & & Entorhinal & 4.702 & 0.077 \\
\hline & & Frontal pole & 1.644 & 0.332 \\
\hline & & Fusiform & 2.222 & 0.246 \\
\hline & & Inferior parietal & 2.952 & 0.170 \\
\hline & & Inferior temporal & 0.001 & 0.987 \\
\hline & & Insula & 0.090 & 0.843 \\
\hline & & Isthmus cingulate & 1.257 & 0.409 \\
\hline & & Lateral occipital & 0.126 & 0.821 \\
\hline & & \begin{tabular}{|l|} 
Lateral orbito frontal \\
\end{tabular} & 0.014 & 0.933 \\
\hline & & Lingual & 5.866 & 0.044 \\
\hline & & Medial orbito frontal & 0.318 & 0.692 \\
\hline & & Middle temporal & 0.097 & 0.842 \\
\hline & & Paracentral & 2.527 & 0.208 \\
\hline & & Parahippocampal & 1.983 & 0.280 \\
\hline & & Pars opercularis & 0.242 & 0.741 \\
\hline & & Pars orbitalis & 0.050 & 0.888 \\
\hline & & Pars triangularis & 0.502 & 0.613 \\
\hline & & Pericalcerine & 2.623 & 0.198 \\
\hline & & Postcentral & 1.806 & 0.306 \\
\hline & & Posterior cingulate & 1.662 & 0.331 \\
\hline
\end{tabular}




\begin{tabular}{|c|c|c|c|c|}
\hline Effect & Side & ROI & $F_{(1,119)}$-value & $\mathrm{p}_{\text {BHadj }}$ \\
\hline & & Precentral & 0.685 & 0.559 \\
\hline & & Precuneus & 2.629 & 0.197 \\
\hline & & Rostral anterior cingulate & 0.453 & 0.628 \\
\hline & & Rostral middle frontal & 0.394 & 0.653 \\
\hline & & Superior frontal & 1.525 & 0.355 \\
\hline & & Superior parietal & 4.186 & 0.096 \\
\hline & & Superior temporal & 0.002 & 0.978 \\
\hline & & Supramarginal & 1.407 & 0.381 \\
\hline & & Temporal pole & 4.445 & 0.087 \\
\hline & & Transverse temporal & 0.024 & 0.923 \\
\hline
\end{tabular}

Table 4. Post-hoc effects of age, sex and NART-IQ on the isotropic signal fraction (ISOSF). $\mathrm{p}_{\mathrm{BHadj}}, 5 \%$ False Discovery Rate Benjamini-Hochberg adjusted $p$ value; ROI, Region of Interest. Significant results are highlighted in bold.

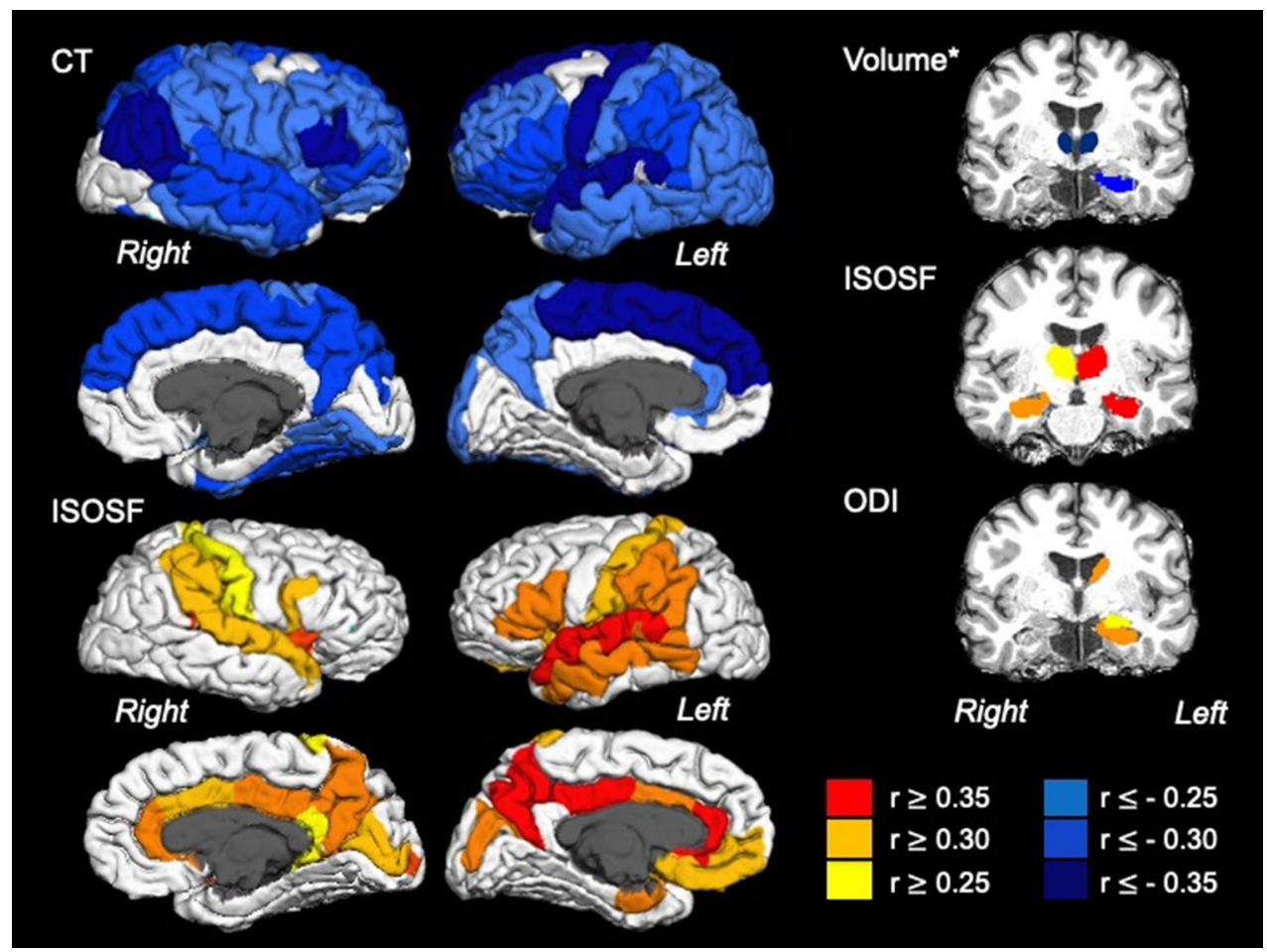

Figure 3. displays the effects of age on cortical thickness (CT), subcortical volume (corrected for intracranial volume), isotropic signal fraction (ISOSF) and orientation dispersion index (ODI) across 34 cortical regions per hemisphere parcellated with the Desikan-Killiany atlas ${ }^{121}$ and seven subcortical regions per hemisphere (hippocampus, amygdala, thalamus, caudate, putamen, globus pallidus, nucleus accumbens). Region of interest segmentations were performed with FreeSurfer (version 5.3). Regions are colour-coded according to the size of the age effect indicated by Pearson correlation coefficient $r$. Warm colours indicate positive and blue colours negative correlations.

While no covariate showed a main effect [systolic BP $(p=0.680)$, diastolic BP $(p=0.750)$, CRP $(p=0.150)$, IL-8 $(p=0.400)$, LAR $(p=0.500)]$, the APOE effect on the left thalamus MPF remained significant $[\mathrm{F}(1,149)=6.7$, $\left.\mathrm{p}_{\mathrm{BHadj}}=0.030\right]$ after accounting for BP measures, but was not significant anymore after controlling for CRP, IL-8 and $\operatorname{LAR}(p=0.060)$. 


\begin{tabular}{|c|c|c|c|c|}
\hline Effect & Side & ROI & $F_{(1,128)}$-value & $\mathbf{p}_{\text {BHadj }}$ \\
\hline \multirow{56}{*}{ Age } & \multirow{7}{*}{ Left } & Accumbens & 3.529 & 0.307 \\
\hline & & Amygdala & 16.646 & $<0.001$ \\
\hline & & \begin{tabular}{|l|} 
Caudate \\
\end{tabular} & 13.995 & $<0.001$ \\
\hline & & Hippocampus & 15.638 & $<0.001$ \\
\hline & & Pallidum & 0.017 & 0.958 \\
\hline & & \begin{tabular}{|l|} 
Putamen \\
\end{tabular} & 3.880 & 0.306 \\
\hline & & Thalamus & 2.111 & 0.505 \\
\hline & \multirow{7}{*}{ Right } & Accumbens & 1.265 & 0.594 \\
\hline & & Amygdala & 7.018 & 0.156 \\
\hline & & Caudate & 0.040 & 0.925 \\
\hline & & Hippocampus & 8.834 & 0.124 \\
\hline & & Pallidum & 0.365 & 0.755 \\
\hline & & \begin{tabular}{|l|} 
Putamen \\
\end{tabular} & 2.142 & 0.506 \\
\hline & & Thalamus & 0.148 & 0.828 \\
\hline & \multirow{31}{*}{ Left } & Banks of superior temporal sulcus & 2.793 & 0.398 \\
\hline & & Caudal anterior cingulate & 7.199 & 0.156 \\
\hline & & Cuneus & 0.001 & 0.992 \\
\hline & & Entorhinal & 5.518 & 0.222 \\
\hline & & Frontal pole & 2.182 & 0.515 \\
\hline & & Fusiform & 2.889 & 0.387 \\
\hline & & Inferior parietal & 0.029 & 0.943 \\
\hline & & Inferior temporal & 1.654 & 0.559 \\
\hline & & Insula & 0.579 & 0.698 \\
\hline & & Lateral occipital & 1.619 & 0.563 \\
\hline & & Lateral orbito frontal & 1.572 & 0.560 \\
\hline & & Lingual & 0.919 & 0.616 \\
\hline & & Medial orbito frontal & 5.107 & 0.253 \\
\hline & & Middle temporal & 1.088 & 0.598 \\
\hline & & Paracentral & 0.634 & 0.693 \\
\hline & & Parahippocampal & 0.173 & 0.826 \\
\hline & & Pars opercularis & 0.076 & 0.892 \\
\hline & & Pars orbitalis & 2.068 & 0.507 \\
\hline & & Pars triangularis & 0.055 & 0.914 \\
\hline & & \begin{tabular}{|l|} 
Postcentral \\
\end{tabular} & 0.526 & 0.705 \\
\hline & & Posterior cingulate & 1.419 & 0.575 \\
\hline & & Precentral & 0.305 & 0.776 \\
\hline & & Precuneus & 0.063 & 0.907 \\
\hline & & Rostral anterior cingulate & 1.459 & 0.576 \\
\hline & & Rostral middle frontal & 2.006 & 0.496 \\
\hline & & Superior frontal & 1.109 & 0.595 \\
\hline & & Superior parietal & 4.078 & 0.326 \\
\hline & & Superior temporal & 2.666 & 0.409 \\
\hline & & Supramarginal & 0.291 & 0.760 \\
\hline & & Temporal pole & 8.362 & 0.130 \\
\hline & & Transverse temporal & 0.200 & 0.817 \\
\hline & \multirow{11}{*}{ Right } & Banks of superior temporal sulcus & 0.534 & 0.712 \\
\hline & & Caudal anterior cingulate & 2.715 & 0.408 \\
\hline & & Cuneus & 0.628 & 0.691 \\
\hline & & Entorhinal & 1.911 & 0.516 \\
\hline & & Frontal pole & 3.977 & 0.312 \\
\hline & & Fusiform & 2.329 & 0.479 \\
\hline & & Inferior parietal & 0.004 & 0.984 \\
\hline & & Inferior temporal & 4.430 & 0.288 \\
\hline & & Insula & 4.760 & 0.268 \\
\hline & & Isthmus cingulate & 5.750 & 0.216 \\
\hline & & Lateral occipital & 1.311 & 0.591 \\
\hline
\end{tabular}




\begin{tabular}{|c|c|c|c|c|}
\hline Effect & Side & ROI & $F_{(1,128)}$-value & $\mathrm{p}_{\text {BHadj }}$ \\
\hline & & Lateral orbito frontal & 1.274 & 0.598 \\
\hline & & Lingual & 0.173 & 0.819 \\
\hline & & Medial orbito frontal & 0.734 & 0.666 \\
\hline & & Middle temporal & 4.509 & 0.295 \\
\hline & & Paracentral & 0.899 & 0.611 \\
\hline & & Parahippocampal & 0.373 & 0.754 \\
\hline & & Pars opercularis & 2.490 & 0.445 \\
\hline & & Pars orbitalis & 1.778 & 0.544 \\
\hline & & Pars triangularis & 0.023 & 0.952 \\
\hline & & Pericalcerine & 0.293 & 0.765 \\
\hline & & Postcentral & 1.564 & 0.553 \\
\hline & & Posterior cingulate & 0.042 & 0.926 \\
\hline & & Precentral & 0.100 & 0.870 \\
\hline & & Precuneus & 0.000 & 0.985 \\
\hline & & Rostral anterior cingulate & 0.284 & 0.760 \\
\hline & & Rostral middle frontal & 0.268 & 0.768 \\
\hline & & Superior frontal & 0.485 & 0.716 \\
\hline & & Superior parietal & 3.130 & 0.352 \\
\hline & & Superior temporal & 5.045 & 0.238 \\
\hline & & Supramarginal & 1.426 & 0.581 \\
\hline & & Temporal pole & 6.156 & 0.198 \\
\hline & & Transverse temporal & 10.589 & 0.039 \\
\hline
\end{tabular}

Table 5. Post-hoc effects of age on the orientation dispersion index (ODI). $\mathrm{p}_{\mathrm{BHadj}}, 5 \%$ False Discovery Rate Benjamini-Hochberg adjusted $p$ value; ROI region of interest. Significant results are highlighted in bold.

\section{Discussion}

Here, we investigated whether qMRI indices of apparent neurite density and dispersion, free water, myelin, and cell metabolism were sensitive to grey matter differences related to LOAD risk in cognitively healthy individuals. Such microstructural measurements hold the potential for novel imaging biomarkers to identify asymptomatic individuals at heightened risk of developing LOAD. As such they may provide non-invasive and cheaper alternatives to PET and cerebrospinal fluid (CSF)-based biomarkers, that are currently employed in clinical trials, in the future.

The only significant difference between asymptomatic $A P O E-\varepsilon 4$ carriers relative to non-carriers was in the qMT measure MPF in the left thalamus with APOE- 44 related reductions in MPF (Fig. 1). This effect was observed independently of age, sex, and verbal intelligence. Reduced MPF may arise from processes that lead to an increase in free water and/or a reduction in the macromolecular content of grey matter including changes in myelin, proteins, and and/or iron concentrations ${ }^{68,69}$. Such changes may be consistent with the presence of inflammatory processes leading to tissue swelling associated with glia activation ${ }^{70}$ and/or with a deficit in cholesterol transport in $A P O E-\varepsilon 4$ carriers ${ }^{70-72}$. Consistent with this interpretation we observed that the effect of APOE genotype on left thalamus MPF was moderated by plasma markers of inflammation (CRP, IL-8, LAR). Furthermore, evidence suggests that $A P O E-\varepsilon 4$ carriage may increase susceptibility to inflammation ${ }^{22,23}$ and that inflammatory processes contribute significantly to the pathogenesis of $\mathrm{LOAD}^{73-75}$.

Notably these APOE- $\varepsilon 4$-related differences in MPF were only observed in the left thalamus but not in any other cortical or subcortical region. The limbic thalamic nuclei maintain dense reciprocal connections with the hippocampal formation and the retrosplenial $\operatorname{cortex}^{76,77}$, which, together with the fornix, mamillary bodies and posterior cingulate cortex, comprise the Papez circuit important for episodic memory function ${ }^{78}$. As outlined above it is increasingly recognised that the Papez circuit, including the anterior thalamus, can be affected early in $\mathrm{LOAD}^{4}$. Neurofibrillary accumulations are found in the anterodorsal thalamic nucleus at the same time as those in the hippocampus in LOAD brains ${ }^{34}$ and neuroimaging studies have revealed reduced thalamic volume in both amnestic $\mathrm{MCI}^{35}$ and LOAD ${ }^{36}$. Furthermore, studies into the effects of APOE in middle-aged asymptomatic adults found reduced glucose metabolism in the thalamus, hippocampus and cingulate cortex ${ }^{39}$ as well as increased metabolism in bilateral thalami and superior temporal gyrus in amyloid- $\beta$ positive $A P O E$ - $\varepsilon 4$ carriers with a maternal history of $\operatorname{LOAD}^{79}$. Cacciaglia et al. ${ }^{80}$ studied the effects of $A P O E$ on grey matter volume in over 500 middle-aged asymptomatic individuals and identified reduced hippocampus, caudate, precentral gyrus, and cerebellum volumes but increased volumes in the thalamus, superior frontal and middle occipital gyri in $A P O E-\varepsilon 4$ carriers. While it remains unknown why $A P O E-\varepsilon 4$ may be related to increased thalamic volume it was suggested that this could reflect brain swelling associated with glial activation in response to larger amyloid- $\beta$ 


\begin{tabular}{|c|c|c|c|c|c|}
\hline Effect & Side & ROI & Index & $F_{(1,149)}$-value & $\mathbf{p}_{\text {BHadj }}$ \\
\hline \multirow{56}{*}{ Age } & \multirow{7}{*}{ Left } & Accumbens & Vol $_{\text {ICVadj }}$ & 7.037 & 0.027 \\
\hline & & Amygdala & Vol ICVadj $_{\text {I }}$ & 3.360 & 0.146 \\
\hline & & Caudate & Vol $_{\text {ICVadj }}$ & 0.073 & 0.873 \\
\hline & & Hippocampus & Vol ICVadj $_{\text {ICl }}$ & 12.023 & 0.004 \\
\hline & & Pallidum & Vol $_{\text {ICVadj }}$ & 1.141 & 0.448 \\
\hline & & \begin{tabular}{|l|} 
Putamen \\
\end{tabular} & Vol $_{\text {ICVadj }}$ & 8.886 & 0.012 \\
\hline & & Thalamus & Vol $_{\text {ICVadj }}$ & 26.144 & $<0.001$ \\
\hline & \multirow{7}{*}{ Right } & Accumbens & Vol ICVadj $_{\text {I }}$ & 4.944 & 0.071 \\
\hline & & Amygdala & Vol ICVadj $_{\text {I }}$ & 3.723 & 0.120 \\
\hline & & Caudate & Vol $_{\text {ICVadj }}$ & 0.225 & 0.778 \\
\hline & & Hippocampus & Vol ICVadj $_{\text {I }}$ & 2.828 & 0.190 \\
\hline & & Pallidum & Vol $_{\text {ICVadj }}$ & 2.444 & 0.221 \\
\hline & & Putamen & Vol ICVadj $_{\text {IC }}$ & 7.722 & 0.021 \\
\hline & & Thalamus & Vol $_{\text {ICVadj }}$ & 45.557 & $<0.001$ \\
\hline & \multirow{34}{*}{ Left } & Banks of superior temporal sulcus & CT & 5.798 & 0.047 \\
\hline & & Caudal anterior cingulate & CT & 0.583 & 0.589 \\
\hline & & Caudal middle frontal & CT & 8.485 & 0.016 \\
\hline & & Cuneus & CT & 3.911 & 0.110 \\
\hline & & Entorhinal & CT & 0.120 & 0.836 \\
\hline & & Frontal pole & CT & 0.076 & 0.885 \\
\hline & & \begin{tabular}{|l|} 
Fusiform \\
\end{tabular} & CT & 5.474 & 0.057 \\
\hline & & Inferior parietal & CT & 11.874 & 0.004 \\
\hline & & Inferior temporal & CT & 7.261 & 0.027 \\
\hline & & Insula & CT & 20.522 & $<0.001$ \\
\hline & & Isthmus cingulate & CT & 0.130 & 0.836 \\
\hline & & Lateral occipital & CT & 4.536 & 0.086 \\
\hline & & \begin{tabular}{|l|} 
Lateral orbito frontal \\
\end{tabular} & CT & 12.478 & 0.006 \\
\hline & & Lingual & CT & 6.891 & 0.030 \\
\hline & & Medial orbito frontal & CT & 7.171 & 0.026 \\
\hline & & Middle temporal & CT & 12.759 & $<0.001$ \\
\hline & & Paracentral & CT & 20.354 & $<0.001$ \\
\hline & & Parahippocampal & CT & 7.647 & 0.022 \\
\hline & & Pars opercularis & CT & 14.469 & $<0.001$ \\
\hline & & Pars orbitalis & CT & 18.893 & $<0.001$ \\
\hline & & Pars triangularis & CT & 19.089 & $<0.001$ \\
\hline & & Pericalcerine & CT & 2.678 & 0.203 \\
\hline & & Postcentral & CT & 12.426 & 0.006 \\
\hline & & Posterior cingulate & CT & 1.032 & 0.467 \\
\hline & & Precentral & CT & 28.246 & $<0.001$ \\
\hline & & Precuneus & CT & 12.353 & 0.006 \\
\hline & & Rostral anterior cingulate & CT & 7.759 & 0.022 \\
\hline & & Rostral middle frontal & CT & 13.280 & $<0.001$ \\
\hline & & Superior frontal & CT & 24.962 & $<0.001$ \\
\hline & & Superior parietal & CT & 9.821 & 0.009 \\
\hline & & Superior temporal & CT & 27.155 & $<0.001$ \\
\hline & & \begin{tabular}{|l|} 
Supramarginal \\
\end{tabular} & CT & 22.159 & $<0.001$ \\
\hline & & Temporal pole & CT & 0.682 & 0.555 \\
\hline & & Transverse temporal & CT & 2.574 & 0.211 \\
\hline & \multirow{8}{*}{ Right } & Banks of superior temporal sulcus & CT & 11.955 & 0.006 \\
\hline & & Caudal anterior cingulate & CT & 3.192 & 0.150 \\
\hline & & Caudal middle frontal & CT & 2.576 & 0.209 \\
\hline & & Cuneus & CT & 1.553 & 0.363 \\
\hline & & Entorhinal & CT & 0.121 & 0.840 \\
\hline & & Frontal pole & CT & 0.015 & 0.938 \\
\hline & & Fusiform & CT & 18.048 & $<0.001$ \\
\hline & & Inferior parietal & CT & 22.640 & $<0.001$ \\
\hline
\end{tabular}




\begin{tabular}{|c|c|c|c|c|c|}
\hline Effect & Side & ROI & Index & $F_{(1,149)}$-value & $\mathbf{p}_{\text {BHadj }}$ \\
\hline & & Inferior temporal & CT & 9.714 & 0.008 \\
\hline & & Insula & CT & 12.353 & 0.005 \\
\hline & & Isthmus cingulate & CT & 4.464 & 0.088 \\
\hline & & Lateral occipital & CT & 4.184 & 0.099 \\
\hline & & \begin{tabular}{|l|} 
Lateral orbito frontal \\
\end{tabular} & CT & 13.295 & $<0.001$ \\
\hline & & Lingual & CT & 7.316 & 0.026 \\
\hline & & Medial orbito frontal & CT & 6.738 & 0.029 \\
\hline & & Middle temporal & CT & 18.517 & $<0.001$ \\
\hline & & Paracentral & CT & 17.110 & $<0.001$ \\
\hline & & Parahippocampal & CT & 8.659 & 0.015 \\
\hline & & Pars opercularis & CT & 12.395 & 0.005 \\
\hline & & Pars orbitalis & CT & 12.59 & 0.005 \\
\hline & & Pars triangularis & CT & 19.087 & $<0.001$ \\
\hline & & Pericalcerine & CT & 2.454 & 0.221 \\
\hline & & \begin{tabular}{|l|} 
Postcentral \\
\end{tabular} & CT & 7.200 & 0.025 \\
\hline & & Posterior cingulate & CT & 6.381 & 0.038 \\
\hline & & Precentral & CT & 10.001 & 0.009 \\
\hline & & Precuneus & CT & 15.729 & $<0.001$ \\
\hline & & Rostral anterior cingulate & CT & 1.949 & 0.290 \\
\hline & & Rostral middle frontal & CT & 10.641 & 0.005 \\
\hline & & Superior frontal & CT & 18.426 & $<0.001$ \\
\hline & & Superior parietal & CT & 7.745 & 0.021 \\
\hline & & Superior temporal & CT & 19.439 & $<0.001$ \\
\hline & & Supramarginal & CT & 10.607 & 0.005 \\
\hline & & Temporal pole & CT & 0.020 & 0.950 \\
\hline & & Transverse temporal & CT & 1.548 & 0.359 \\
\hline \multirow{30}{*}{ Sex } & \multirow{7}{*}{ Left } & Accumbens & Vol ICVadj $_{\text {I }}$ & 8.927 & 0.012 \\
\hline & & Amygdala & Vol $_{\text {ICVadj }}$ & 0.074 & 0.878 \\
\hline & & Caudate & Vol $_{\text {ICVadj }}$ & 4.492 & 0.086 \\
\hline & & Hippocampus & Vol $_{\text {ICVadj }}$ & 10.913 & 0.007 \\
\hline & & Pallidum & Vol ICVadj $_{\text {I }}$ & 1.649 & 0.343 \\
\hline & & \begin{tabular}{|l|} 
Putamen \\
\end{tabular} & Vol ICVadj $_{\text {ICl }}$ & 6.103 & 0.042 \\
\hline & & \begin{tabular}{|l|} 
Thalamus \\
\end{tabular} & Vol $_{\text {ICVadj }}$ & 1.934 & 0.289 \\
\hline & \multirow{7}{*}{ Right } & Accumbens & Vol $_{\text {ICVadj }}$ & 3.833 & 0.113 \\
\hline & & Amygdala & Vol $_{\text {ICVadj }}$ & 0.513 & 0.623 \\
\hline & & Caudate & Vol ICVadj $_{\text {I }}$ & 7.183 & 0.025 \\
\hline & & Hippocampus & Vol $_{\text {ICVadj }}$ & 4.695 & 0.080 \\
\hline & & \begin{tabular}{|l|} 
Pallidum \\
\end{tabular} & Vol ICVadj $_{\text {ICl }}$ & 7.633 & 0.020 \\
\hline & & Putamen & Vol $_{\text {ICVadj }}$ & 4.265 & 0.096 \\
\hline & & Thalamus & Vol $_{\text {ICVadj }}$ & 4.360 & 0.090 \\
\hline & \multirow{16}{*}{ Left } & Banks of superior temporal sulcus & CT & 3.183 & 0.157 \\
\hline & & Caudal anterior cingulate & CT & 0.019 & 0.935 \\
\hline & & Caudal middle frontal & CT & 0.018 & 0.934 \\
\hline & & Cuneus & CT & 1.857 & 0.302 \\
\hline & & Entorhinal & CT & 0.075 & 0.881 \\
\hline & & Frontal pole & CT & 0.794 & 0.519 \\
\hline & & Fusiform & CT & 0.285 & 0.761 \\
\hline & & Inferior parietal & CT & 2.104 & 0.268 \\
\hline & & Inferior temporal & CT & 0.229 & 0.780 \\
\hline & & Insula & CT & 9.485 & 0.008 \\
\hline & & Isthmus cingulate & CT & 0.031 & 0.928 \\
\hline & & Lateral occipital & CT & 0.244 & 0.772 \\
\hline & & Lateral orbito frontal & CT & 0.058 & 0.886 \\
\hline & & Lingual & CT & 0.891 & 0.503 \\
\hline & & Medial orbito frontal & CT & 1.146 & 0.455 \\
\hline & & Middle temporal & CT & 0.206 & 0.783 \\
\hline
\end{tabular}




\begin{tabular}{|c|c|c|c|c|c|}
\hline Effect & Side & ROI & Index & $F_{(1,149)}$-value & $\mathbf{p}_{\text {BHadj }}$ \\
\hline & & Paracentral & CT & 2.266 & 0.244 \\
\hline & & Parahippocampal & CT & 0.936 & 0.490 \\
\hline & & Pars opercularis & CT & 1.245 & 0.436 \\
\hline & & Pars orbitalis & CT & 0.134 & 0.837 \\
\hline & & Pars triangularis & CT & 2.647 & 0.204 \\
\hline & & Pericalcerine & CT & 0.202 & 0.782 \\
\hline & & Postcentral & CT & 4.122 & 0.100 \\
\hline & & Posterior cingulate & CT & 0.295 & 0.759 \\
\hline & & Precentral & CT & 0.008 & 0.948 \\
\hline & & Precuneus & CT & 0.098 & 0.859 \\
\hline & & Rostral anterior cingulate & CT & 0.038 & 0.917 \\
\hline & & Rostral middle frontal & CT & 0.019 & 0.941 \\
\hline & & Superior frontal & CT & 1.171 & 0.451 \\
\hline & & Superior parietal & CT & 0.459 & 0.649 \\
\hline & & Superior temporal & CT & 0.141 & 0.835 \\
\hline & & Supramarginal & CT & 4.028 & 0.105 \\
\hline & & Temporal pole & CT & 1.133 & 0.447 \\
\hline & & Transverse temporal & CT & 1.466 & 0.377 \\
\hline & \multirow{34}{*}{ Right } & Banks of superior temporal sulcus & CT & 3.084 & 0.166 \\
\hline & & Caudal anterior cingulate & CT & 0.069 & 0.872 \\
\hline & & Caudal middle frontal & CT & 0.809 & 0.527 \\
\hline & & Cuneus & CT & 0.855 & 0.513 \\
\hline & & Entorhinal & CT & 0.746 & 0.536 \\
\hline & & Frontal pole & CT & 1.243 & 0.433 \\
\hline & & Fusiform & CT & 0.799 & 0.522 \\
\hline & & Inferior parietal & CT & 5.173 & 0.063 \\
\hline & & Inferior temporal & CT & 0.019 & 0.946 \\
\hline & & Insula & CT & 5.346 & 0.059 \\
\hline & & Isthmus cingulate & CT & 6.254 & 0.037 \\
\hline & & Lateral occipital & CT & 0.625 & 0.574 \\
\hline & & Lateral orbito frontal & CT & 2.769 & 0.193 \\
\hline & & Lingual & CT & 0.267 & 0.770 \\
\hline & & Medial orbito frontal & CT & 0.941 & 0.493 \\
\hline & & Middle temporal & CT & 0.167 & 0.811 \\
\hline & & Paracentral & CT & 2.089 & 0.267 \\
\hline & & Parahippocampal & CT & 1.127 & 0.444 \\
\hline & & Pars opercularis & CT & 0.993 & 0.478 \\
\hline & & Pars orbitalis & CT & 0.670 & 0.556 \\
\hline & & Pars triangularis & CT & 0.007 & 0.944 \\
\hline & & Pericalcerine & CT & 0.008 & 0.959 \\
\hline & & Postcentral & CT & 2.954 & 0.178 \\
\hline & & Posterior cingulate & CT & 0.704 & 0.550 \\
\hline & & Precentral & CT & 0.252 & 0.771 \\
\hline & & Precuneus & CT & 0.806 & 0.524 \\
\hline & & Rostral anterior cingulate & CT & 1.115 & 0.444 \\
\hline & & Rostral middle frontal & CT & 0.008 & 0.953 \\
\hline & & Superior frontal & CT & 0.003 & 0.959 \\
\hline & & Superior parietal & CT & 4.903 & 0.072 \\
\hline & & Superior temporal & CT & 0.220 & 0.777 \\
\hline & & Supramarginal & CT & 1.145 & 0.451 \\
\hline & & Temporal pole & CT & 0.005 & 0.951 \\
\hline & & Transverse temporal & CT & 0.262 & 0.768 \\
\hline
\end{tabular}

Table 6. Post-hoc effects of age and sex on cortical thickness and subcortical volume measures. CT cortical thickness; Vol $_{\text {ICVadj }}$ volume adjusted for intracranial volume. $\mathrm{p}_{\mathrm{BHadj}}, 5 \%$ False Discovery Rate BenjaminiHochberg adjusted $p$ value; ROI region of interest. 
burden $^{81}$. As mentioned above, the here observed pattern of APOE- $\varepsilon 4$-related reductions in MPF in the left thalamus is consistent with this interpretation ${ }^{56,82}$. One other study investigated the impact of $A P O E-\varepsilon 4$ on qMT white matter metrics in young adults and did not find any differences ${ }^{83}$. This suggests that such risk-related glial dysfunction may accumulate with age and may only become apparent from midlife onwards.

The question arises why we did not observe any risk-related effects in brain regions that have previously been reported to be affected by LOAD risk factors ${ }^{10,84,85}$. Reports with regards to the impact of APOE- $\varepsilon 4$ on grey matter structures in healthy young and middle-aged adults have been mixed ${ }^{10,84}$, with some studies reporting no changes in hippocampal grey matter volume in $A P O E-\varepsilon 4$ carriers $^{31,86}$. Studies assessing the impact of $A P O E-\varepsilon 4$ on tissue microstructure have primarily focused on diffusion tensor imaging (DTI) of white matter. While some reported widespread white matter differences in DTI measures ${ }^{83,87,88}$, this has not been replicated in all studies ${ }^{30,89}$. These discrepancies may arise due to DTI indices not being sufficiently sensitive and/or specific to detect early risk-related tissue abnormalities ${ }^{90}$. Direct comparisons between DTI and NODDI indices revealed that although fractional anisotropy (FA) was sensitive to white matter differences between healthy controls and patients with metabolic disease, FA was less anatomically specific and did not identify all brain regions that were captured by ICSF and ODI ${ }^{91}$. Thus we employed NODDI and qMT measurements to study risk effects on grey matter here and on white matter in a previous CARDS analysis ${ }^{92}$. In the previously published white matter analysis ${ }^{92}$ we did not observe any main effects of risk but found that individuals with the highest genetic risk (obese $\mathrm{FH}+$ and $A P O E-\varepsilon 4$ ) exhibited obesity-related reductions in MPF and ICSF in the right parahippocampal cingulum.

Taken together, our previous and here reported findings demonstrate that MPF from qMT can identify risk-related microstructural differences in limbic grey and white matter that were not apparent in conventional volumetric or cortical thickness measurements. We propose that these differences may reflect subtle changes related to neuroglia activation and that limbic structures including the thalamus are particularly susceptible to adverse effects of $A P O E-\varepsilon 4$ on glia cells. Inconsistencies in previous studies may have arisen from standard morphological and DTI measurements not being sensitive and/or specific enough to detect such glia-related changes.

It is important to note that while we did not find any risk-related effects on brain morphology we did replicate the well-established pattern of widespread age-related thinning in frontal, temporal and parietal regions ${ }^{93}$ as well as volume loss in subcortical structures including the hippocampi and thalami (Fig. 3). The subcortical volume loss was accompanied by age-related increases in ISOSF in bilateral hippocampi and thalami but effects on cortical regions were more localised: increased ISOSF was apparent along medial regions of the cingulate and parietal cortices including the precuneus as well as in superior temporal and lateral and orbito prefrontal cortices. Age-related increases in ISOSF have been previously observed ${ }^{94}$ and most likely reflect lost tissue being replaced by CSF. Consistent with a previous study ${ }^{95}$ we also observed a positive correlation between age and ODI, an estimate of neurite dispersion, in the hippocampus and the left caudate and amygdala. In contrast to Nazari et al. ${ }^{95}$ however, we did not find any effects in cortical regions, while they reported reduced ODI with age in fronto-parietal regions. These opposing patterns in cortical and subcortical regions may reflect age-related reductions of neocortical dendritic spine density ${ }^{96}$ with accompanying compensatory increases in the dendritic extent of dentate gyrus granular cells ${ }^{97,98}$. Similar age-related increases in the dendritic tree have also been reported in the basolateral nucleus of the amygdala of rats ${ }^{99}$.

Furthermore, we observed positive correlations between ISOSF and NART-IQ in superior temporal, parietal and lingual cortices that were partly driven by age. NART requires the reading of irregularly pronounced words and older relative to younger adults tended to perform better in the NART. However, positive albeit weak correlations between NART-IQ and ISOSF remained for the left superior temporal sulcus and left superior parietal cortex. Developmental imaging studies have revealed cortical thinning during adolescence ${ }^{100}$ that may be due to increased myelination ${ }^{100}$ or synaptic pruning and dendritic arborization ${ }^{101,102}$. It may therefore be possible that childhood developmental differences in cortical maturation as well as in education may have contributed to this effect. For instance, childhood cognitive abilities have been found to account for relationships between cognitive performance and brain cortical thickness decades later in older adults from the Lothian birth cohort ${ }^{103}$.

Consistent with previous reports ${ }^{104}$ we did not observe widespread sex-differences in brain morphology measurements with the exception of larger volumes in the left hippocampus in women than men ${ }^{105}$. However, qMRI indices revealed the following pattern: Women compared to men, had lower ISOSF in widespread cortical and subcortical regions and larger MPF in frontal and temporal regions. Previously we also reported higher MPF and lower ISOSF for white matter in women than $\mathrm{men}^{44}$. Overall this pattern of sex differences suggests higher cortical myelination and lower free water signal in women as they tended to be overall in better health i.e. were less obese, had lower systolic BP, and reported drinking less alcohol than men ${ }^{44}$. All of these factors may have contributed to women showing "healthier" grey and white matter in the CARDS cohort.

Finally, some study limitations need to be considered. First of all, CARDS is a cross-sectional study that cannot answer whether the observed APOE effects on left thalamus MPF are predictive of accelerated development of LOAD pathology, cognitive, or neuronal decline. Future prospective longitudinal studies are required to address this question. We also propose that our findings require replication in larger samples that can control for possible interactions between APOE and other LOAD risk genes such as variants of TREM2 and polygenic risk hazards as the number of participants in the CARDS study was too small to do so. It is also worth mentioning that other qMRI measurements, that were not included in the current study, may prove helpful in characterising risk effects on the brain. Notably quantitative $\mathrm{T}_{2}$ and $\mathrm{T}_{2}{ }^{*}$ measurements have been proposed to be sensitive to neurodegenerative processes. For instance, prolonged $\mathrm{T}_{2}$ relaxometry has been reported in the hippocampus of LOAD patients ${ }^{106}$ and has been proposed to increase the sensitivity and specificity of MCI and LOAD detection ${ }^{107}$. Finally, it should be noted that we only studied the thalamus as a whole structure while neuropathological evidence suggests a specific vulnerability of the anterodorsal thalamic nucleus to LOAD pathology. Future studies may investigate 
risk-related effects on specific subthalamic nuclei, which was beyond the scope of the current study as we were focusing on risk effects across the whole brain.

In summary, we have shown $A P O E-\varepsilon 4$ related reductions in the qMT measure MPF in the left thalamus that were moderated by peripheral markers of inflammation. This effect occurred independently of age, sex and NART-IQ and was not observed in morphological or microstructural indices from diffusion-weighted imaging. In addition, the effect was specific to the left thalamus and was not present in other cortical and subcortical grey matter regions. We propose that MPF reductions may reflect the effects of glia-mediated inflammatory and demyelination processes in $A P O E-\varepsilon 4$ carriers. As such qMT measurements hold the potential for non-invasive and cheaper biomarker alternatives to PET, that may aid our understanding of the pathological processes leading to LOAD. In addition, qMT may help with the identification of asymptomatic individuals at heightened risk of LOAD for stratification into clinical trials for future preventative therapeutics.

\section{Materials and methods}

The Cardiff Ageing and Risk of Dementia Study (CARDS) has been described previously including a detailed description of the participant sample ${ }^{43,92}$, assessment of genetic and metabolic risk factors ${ }^{44,92}$ and the acquisition and processing of the MRI data ${ }^{43,44,92,108}$. Here we provide a brief summary of the most important points. CARDS received ethical approval from the School of Psychology Research Ethics Committee at Cardiff University (EC.14.09.09.3843R2) and all participants provided written informed consent in accordance with the Declaration of Helsinki. All research methods were performed in line with Cardiff University's Research Integrity and Governance Code of Practice and relevant data protection regulations.

Participants. The CARDS cohort comprised 166 community-dwelling individuals between the age of 38 and 71 years who underwent cognitive and health assessment as well as MRI scanning (Table 1). Exclusion criteria were a history of neurological and/or psychiatric disease, head injury, drug/alcohol dependency, high risk cardio-embolic source, large-vessel disease or MRI incompatibility due to pacemaker, stents or other surgical implants. As a group, participants intellectual functioning was above average as assessed with the National Adult Reading Test (NART) ${ }^{66}$. All but one participant scored $>26$ on the Mini Mental State Exam (MMSE) ${ }^{42}$ thus the remaining 165 participants were classified as cognitively healthy. Eight participants scored $\geq 10$ in the Patient Health Questionnaire (PHQ)-9 ${ }^{109}$, suggesting moderate levels of depression but no participant was severely depressed.

Assessment of risk factors. Saliva samples were collected with the Genotek Oragene-DNA kit (OG-500) and $A P O E$ genotypes $\varepsilon 2, \varepsilon 3$, and $\varepsilon 4$ were determined with TaqMan genotyping of single nucleotide polymorphism (SNP) rs7412 and KASP genotyping of SNP rs429358. Participants self-reported their family history of dementia, i.e., whether a first-grade relative was affected by Alzheimer's disease, vascular dementia or any other type of dementia.

Central obesity was assessed from the waist-hip ratio (WHR) ${ }^{44}$ with abdominal obesity defined as a WHR $\geq 0.9$ for males and $\geq 0.85$ for females. Resting systolic and diastolic blood pressure (BP) readings were taken with a digital blood pressure monitor (Model UA-631; A\&D Medical, Tokyo, Japan) and the means of three readings were calculated. Participants self-reported other metabolic risk factors, including diabetes mellitus, high levels of blood cholesterol controlled with statin medication, history of smoking, and weekly alcohol intake. There were only few diabetics, smokers, and individuals on statins and, hence, these variables were not included in the analyses.

Blood plasma analysis. As previously reported ${ }^{44,92}$, venous blood samples were drawn into 9 ml heparin coated plasma tubes after $12 \mathrm{~h}$ overnight fasting and were centrifuged for $10 \mathrm{~min}$ at $2000 \times \mathrm{g}$ within $1 \mathrm{~h}$ from blood collection. Plasma samples were then transferred into $0.5 \mathrm{ml}$ polypropylene microtubes and stored in a freezer at $-80^{\circ} \mathrm{C}$. Circulating levels of high-sensitivity C-Reactive Protein (CRP) in $\mathrm{mg} / \mathrm{dL}$ were assayed using a human CRP Quantikine enzyme-linked immunosorbent assay (ELISA) kit (R \& D Systems, Minneapolis, USA). Six individuals had a CRP value $>10 \mathrm{mg} / \mathrm{ml}$ indicative of acute infection and were, therefore, excluded from the statistical analyses testing for moderating effects of inflammation. Leptin concentrations in $\mathrm{pg} / \mathrm{ml}$ were determined with the DRP300 Quantikine ELISA kit (R \& D Systems) and adiponectin in $\mathrm{ng} / \mathrm{ml}$ with the human total adiponectin/Acrp30 Quantitkine ELISA kit (R \& D Systems). Leptin/adiponectin ratios for each participant were calculated. Interleukin IL-8 levels in $\mathrm{pg} / \mathrm{mL}$ were determined using a high sensitivity CXCL8/ INTERLEUKIN-8 Quantikine ELISA kit (R \& D Systems). Determination of interleukin-1 $\beta$, interleukin-6 and Tumor Necrosis Factor a (TNFa) levels were trialled with high-sensitivity Quantikine ELISA kits but did not result in reliable measurements consistently above the level of detection for each assay.

MRI data acquisition. MRI data were acquired on a 3 T MAGNETOM Prisma clinical scanner (Siemens Healthcare, Erlangen, Germany) as described in ${ }^{43,44,92,108}$. $\mathrm{T}_{1}$-weighted images $(1 \times 1 \times 1 \mathrm{~mm}$ voxel $)$ were collected with a three-dimension (3D) magnetization-prepared rapid gradient-echo (MP-RAGE) sequence $(256 \times 256$ acquisition matrix, $\mathrm{TR}=2300 \mathrm{~ms}, \mathrm{TE}=3.06 \mathrm{~ms}, \mathrm{TI}=850 \mathrm{~ms}$, flip angle $\theta=9^{\circ}, 176$ slices, $1 \mathrm{~mm}$ slice thickness, $\mathrm{FOV}=256 \mathrm{~mm}$ and acquisition time of $\sim 6 \mathrm{~min}$ ).

High Angular Resolution Diffusion Imaging (HARDI $)^{51}$ data $(2 \times 2 \times 2 \mathrm{~mm}$ voxel) were collected with a spin-echo echo-planar dual shell HARDI sequence with diffusion encoded along 90 isotropically distributed orientations ${ }^{110}$ (30 directions at $b$-value $=1200 \mathrm{~s} / \mathrm{mm}^{2}$ and 60 directions at $b$-value $=2400 \mathrm{~s} / \mathrm{mm}^{2}$ ) and six nondiffusion weighted scans with dynamic field correction and the following parameters: $\mathrm{TR}=9400 \mathrm{~ms}, \mathrm{TE}=67 \mathrm{~ms}$, 
80 slices, $2 \mathrm{~mm}$ slice thickness, FOV $=256 \times 256 \times 160 \mathrm{~mm}$, GRAPPA acceleration factor $=2$ and acquisition time of $\sim 15 \mathrm{~min}$.

Quantitative magnetization transfer weighted imaging (qMT) data were acquired with a prototype sequence, i.e. an optimized 3D MT-weighted gradient-recalled-echo sequence ${ }^{46}$ to obtain magnetization transfer-weighted data with the following parameters: $\mathrm{TR}=32 \mathrm{~ms}, \mathrm{TE}=2.46 \mathrm{~ms}$; Gaussian MT pulses, duration $\mathrm{t}=12.8 \mathrm{~ms} ; \mathrm{FA}=5^{\circ}$; FOV $=24 \mathrm{~cm}, 2.5 \times 2.5 \times 2.5 \mathrm{~mm}^{3}$ resolution. The following off-resonance irradiation frequencies $(\Theta)$ and their corresponding saturation pulse nominal flip angles $(\triangle \mathrm{SAT})$ for the $11 \mathrm{MT}$-weighted images were optimized using Cramer-Rao lower bound optimization: $\Theta=[1000 \mathrm{~Hz}, 1000 \mathrm{~Hz}, 2750 \mathrm{~Hz}, 2768 \mathrm{~Hz}, 2790 \mathrm{~Hz}, 2890 \mathrm{~Hz}, 1000 \mathrm{~Hz}$, $1000 \mathrm{~Hz}, 12,060 \mathrm{~Hz}, 47,180 \mathrm{~Hz}, 56,360 \mathrm{~Hz}]$ and their corresponding $\Delta S A T$ values $=\left[332^{\circ}, 333^{\circ}, 628^{\circ}, 628^{\circ}, 628^{\circ}\right.$, $628^{\circ}, 628^{\circ}, 628^{\circ}, 628^{\circ}, 628^{\circ}, 332^{\circ}$. The longitudinal relaxation time, $\mathrm{T}_{1}$, of the system was estimated by acquiring three $3 \mathrm{D}$ gradient recalled echo sequence (GRE) volumes with three different flip angles $\left(\theta=3^{\circ}, 7^{\circ}, 15^{\circ}\right)$ using the same acquisition parameters as used in the MT-weighted sequence $(\mathrm{TR}=32 \mathrm{~ms}, \mathrm{TE}=2.46 \mathrm{~ms}, \mathrm{FOV}=24 \mathrm{~cm}$, $2.5 \times 2.5 \times 2.5 \mathrm{~mm}^{3}$ resolution). Data for computing the static magnetic field $\left(\mathrm{B}_{0}\right)$ were collected using two $3 \mathrm{D}$ GRE volumes with different echo-times $(\mathrm{TE}=4.92 \mathrm{~ms}$ and $7.38 \mathrm{~ms}$ respectively; $\mathrm{TR}=330 \mathrm{~ms} ; \mathrm{FOV}=240 \mathrm{~mm}$; slice thickness $2.5 \mathrm{~mm})^{111}$. The acquisition time for the complete qMT sequence including all fieldmaps was $\sim 30 \mathrm{~min}$.

HARDI and qMT data processing. As described in ${ }^{43,44,92,108}$, the dual-shell HARDI data were split and $\mathrm{b}=1200$ and $2400 \mathrm{~s} / \mathrm{mm}^{2}$ data were corrected separately for distortions induced by the diffusion-weighted gradients and motion artifacts with appropriate reorientation of the encoding vectors ${ }^{12}$ in ExploreDTI (Version 4.8.3 $)^{113}$. EPI-induced geometrical distortions were corrected by warping the diffusion-weighted image volumes to the $\mathrm{T}_{1}$-weighted anatomical images ${ }^{114}$. After pre-processing, the NODDI model ${ }^{45}$ was fitted to the HARDI data with the fast, linear model fitting algorithms of the Accelerated Microstructure Imaging via Convex Optimization (AMICO) framework ${ }^{115}$ to gain ISOSF, ICSF, and ODI maps.

Using Elastix ${ }^{116}$, MT-weighted GRE volumes were co-registered to the MT-volume with the most contrast using a rigid body ( 6 degrees of freedom) registration to correct for inter-scan motion. Data from the 11 MT-weighted GRE images and $\mathrm{T}_{1}$-maps were fitted by a two-pool model using the Ramani pulsed-MT approximation $^{117}$. This approximation provided MPF and $k_{f}$ maps. To remove voxels with noise-only data, MPF maps were thresholded to an upper intensity limit of 0.3 and $k_{f}$ maps to an upper limit of 3.0 using the fslmaths imaging calculator from the Functional Magnetic Resonance Imaging of the Brain (FMRIB) library (version 6).

All image modality maps were spatially aligned to the $\mathrm{T}_{1}$-weighted anatomical volume as reference image with linear affine registration (12 degrees of freedom) in within-subject space using FMRIB's Linear Image Registration Tool (FLIRT) ${ }^{118,119}$.

Cortical and subcortical grey matter region segmentation. Grey matter cortical and subcortical regions were automatically segmented from $\mathrm{T}_{1}$ - weighted images with the Freesurfer image analysis suite (version 5.3), which is documented online (https://surfer.nmr.mgh.harvard.edu/) ${ }^{64}$. The images were processed by running the "recon-all" script using the default analysis settings. In brief, the images were registered to the Montreal Neurological Institute standard space and intensity normalization was performed. This was followed by automatic skull stripping to remove extracerebral structures, the cerebellum and the brain stem, followed by segmentation into grey matter, white matter and CSF and separation of the hemispheres. Pial surfaces were obtained by tessellating the grey and white matter boundary and by surface deformation following intensity gradients for optimal placement of grey and white matter and grey matter and CSF boundaries ${ }^{120}$. Surface inflation and registration to a spherical atlas were then performed and the cerebral cortex was parcellated into 34 regions per hemisphere based on gyral and sulcal structures following the Desikan-Killiany atlas ${ }^{121}$. Cortical thickness measurements were estimated as the average shortest distance between the pial surface and the white matter boundary ${ }^{122}$. For each hemisphere, seven deep grey matter structures (hippocampus, amygdala, thalamus, caudate, putamen, pallidum, and nucleus accumbens) were automatically parcellated using a probabilistic atlas so that average volumetric measurements could be determined ${ }^{123,124}$. Mean intracranial volume fractions (ICV) were extracted for each brain as estimates of individual differences in head sizes and all volumetric measurements were adjusted for ICV by dividing each participant's subcortical volume by their ICV.

Finally, the mean values of all microstructural indices were extracted from each participants' cortical and subcortical region of interests. Mean measurements were taken in each participants' native space. This was done by first converting each participants' cortical and subcortical masks from the FreeSurfer Massachusetts General Hospital volume file format (MGZ) into the Neuroimaging Informations Technology Initiative (NIfTI) analyze-style data format and then uploading the microstructural maps onto each region of interest mask using the fslmaths command from the FMRIB library. Mean values of each index for each mask were then extracted using the FMRIB fslstats command. NODDI and qMT indices of ISOSF, ICSF, ODI, MPF and $k_{f}$, could not be extracted from bilateral caudal middle frontal, left isthmus cingulate and left pericalcarine regions and $\mathrm{R}_{1}$ could not be extracted from the right postcentral region.

Received: 27 July 2020; Accepted: 15 October 2020

Published online: 13 November 2020

\section{References}

1. World Health Organisation. Dementia Factsheet. (https://www.who.int/news-room/fact-sheets/detail/dementia, 2019).

2. Braak, H. \& Del Trecidi, K. Neuroanatomy and pathology of sporadic Alzheimer's disease. Adv. Anat. Embryol. Cell Biol. 215, $1-162(2015)$. 
3. Braak, H. \& Del Tredici, K. The preclinical phase of the pathological process underlying sporadic Alzheimer's disease. Brain 138, 2814-2833 (2015).

4. Aggleton, J. P., Pralus, A., Nelson, A. J. \& Hornberger, M. Thalamic pathology and memory loss in early Alzheimer's disease: moving the focus from the medial temporal lobe to Papez circuit. Brain 139, 1877-1890 (2016).

5. Jack, C. R. et al. Tracking pathophysiological processes in Alzheimer's disease: an updated hypothetical model of dynamic biomarkers. Lancet Neurol. 12, 207-216 (2013).

6. Hersi, M. et al. Risk factors associated with the onset and progression of Alzheimer's disease: a systematic review of the evidence. Neurotoxicology 61, 143-187 (2017).

7. Mahoney-Sanchez, L., Belaidi, A. A., Bush, A. I. \& Ayton, S. The complex role of apolipoprotein E in Alzheimer's disease: an overview and update. J. Mol. Neurosci. 60, 325-335 (2016).

8. Liu, C. C., Kanekiyo, T., Xu, H. \& Bu, G. Apolipoprotein E and Alzheimer disease: risk, mechanisms and therapy. Nat. Rev. Neurol. 9, 106-118 (2013).

9. Filippini, N. et al. Differential effects of the APOE genotype on brain function across the lifespan. Neuroimage 54, 602-610 (2011).

10. Chételat, G. \& Fouquet, M. Neuroimaging biomarkers for Alzheimer's disease in asymptomatic APOE4 carriers. Rev. Neurol. (Paris) 169, 729-736 (2013).

11. Gottesman, R. F. et al. The ARIC-PET amyloid imaging study: Brain amyloid differences by age, race, sex, and APOE. Neurology 87, 473-480 (2016).

12. Kantarci, K. et al. APOE modifies the association between Abeta load and cognition in cognitively normal older adults. Neurology 78, 232-240 (2012).

13. Lim, Y. Y. et al. Effect of APOE genotype on amyloid deposition, brain volume, and memory in cognitively normal older individuals. J. Alzheimers Dis. 58, 1293-1302 (2017).

14. Toledo, J. B. et al. APOE effect on amyloid-beta PET spatial distribution, deposition rate, and cut-points. J. Alzheimers Dis. 69, 783-793 (2019).

15. Yi, D. et al. Synergistic interaction between APOE and family history of Alzheimer's disease on cerebral amyloid deposition and glucose metabolism. Alzheimers. Res. Ther. 10, 84 (2018).

16. Payami, H. et al. A prospective study of cognitive health in the elderly (Oregon Brain aging study): effects of family history and apolipoprotein E genotype. Am. J. Hum. Genet. 60, 948-956 (1997).

17. Kunkle, B. W. et al. Genetic meta-analysis of diagnosed Alzheimer's disease identifies new risk loci and implicates Abeta, tau, immunity and lipid processing. Nat. Genet. 51, 414-430 (2019).

18. Marioni, R. E. et al. GWAS on family history of Alzheimer's disease. Transl. Psychiatry. 8, 99 (2018).

19. Chuang, Y. F. et al. Midlife adiposity predicts earlier onset of Alzheimer's dementia, neuropathology and presymptomatic cerebral amyloid accumulation. Mol. Psychiatry 21, 910-915 (2016).

20. Pedditizi, E., Peters, R. \& Beckett, N. The risk of overweight/obesity in mid-life and late life for the development of dementia: a systematic review and meta-analysis of longitudinal studies. Age Ageing 45, 14-21 (2016).

21. Cox, A. J., West, N. P. \& Cripps, A. W. Obesity, inflammation, and the gut microbiota. Lancet Diabetes Endocrinol. 3, 207-215 (2015).

22. Ghebranious, N. et al. A pilot study of gene/gene and gene/environment interactions in Alzheimer disease. Clin. Med. Res. 9, 17-25 (2011).

23. Jones, N. S. \& Rebeck, G. W. The synergistic effects of APOE genotype and obesity on Alzheimer's disease risk. Int J Mol Sci 20, $63(2018)$

24. Donix, M. et al. Family history of Alzheimer's disease and hippocampal structure in healthy people. Am. J. Psychiatry 167, 1399-1406 (2010).

25. Reiter, K. et al. Five-year longitudinal brain volume change in healthy elders at genetic risk for Alzheimer's disease. J. Alzheimers Dis. 55, 1363-1377 (2017).

26. Tardif, C. L. et al. Regionally specific changes in the hippocampal circuitry accompany progression of cerebrospinal fluid biomarkers in preclinical Alzheimer's disease. Hum. Brain. Mapp. 39, 971-984 (2018).

27. McKhann, G. M. et al. The diagnosis of dementia due to Alzheimer's disease: recommendations from the National Institute on Aging-Alzheimer's Association workgroups on diagnostic guidelines for Alzheimer's disease. Alzheimers Dement. 7, 263-269 (2011).

28. Falgàs, N. et al. Hippocampal atrophy has limited usefulness as a diagnostic biomarker on the early onset Alzheimer's disease patients: A comparison between visual and quantitative assessment. Neuroimage Clin. 23, 101927 (2019).

29. Halliday, G. Pathology and hippocampal atrophy in Alzheimer's disease. Lancet Neurol. 16, 862-864 (2017).

30. Reinvang, I., Espeseth, T. \& Westlye, L. T. APOE-related biomarker profiles in non-pathological aging and early phases of Alzheimer's disease. Neurosci. Biobehav. Rev. 37, 1322-1335 (2013).

31. Khan, W. et al. No differences in hippocampal volume between carriers and non-carriers of the ApoE $\varepsilon 4$ and $\varepsilon 2$ alleles in young healthy adolescents. J. Alzheimers Dis. 40, 37-43 (2014).

32. de Flores, R., La Joie, R. \& Chételat, G. Structural imaging of hippocampal subfields in healthy aging and Alzheimer's disease. Neuroscience 309, 29-50 (2015).

33. Kerchner, G. A. et al. APOE epsilon4 worsens hippocampal CA1 apical neuropil atrophy and episodic memory. Neurology 82, 691-697 (2014).

34. Braak, H. \& Braak, E. Alzheimer's disease affects limbic nuclei of the thalamus. Acta Neuropathol. 81, 261-268 (1991).

35. Chételat, G. et al. Using voxel-based morphometry to map the structural changes associated with rapid conversion in MCI: a longitudinal MRI study. Neuroimage 27, 934-946 (2005).

36. Cherubini, A. et al. Combined volumetry and DTI in subcortical structures of mild cognitive impairment and Alzheimer's disease patients. J. Alzheimers Dis. 19, 1273-1282 (2010).

37. Ryan, N. S. et al. Magnetic resonance imaging evidence for presymptomatic change in thalamus and caudate in familial Alzheimer's disease. Brain 136, 1399-1414 (2013).

38. Paranjpe, M. D. et al. The effect of ApoE $\varepsilon 4$ on longitudinal brain region-specific glucose metabolism in patients with mild cognitive impairment: a FDG-PET study. Neuroimage Clin. 22, 101795 (2019).

39. Langbaum, J. B. et al. Hypometabolism in Alzheimer-affected brain regions in cognitively healthy Latino individuals carrying the apolipoprotein E epsilon4 allele. Arch. Neurol. 67, 462-468 (2010).

40. Valla, J. et al. Reduced posterior cingulate mitochondrial activity in expired young adult carriers of the APOE $\varepsilon 4$ allele, the major late-onset Alzheimer's susceptibility gene. J. Alzheimers Dis. 22, 307-313 (2010).

41. Matsuda, H., Shigemoto, Y. \& Sato, N. Neuroimaging of Alzheimer's disease: focus on amyloid and tau PET. Jpn. J. Radiol. 37, 735-749 (2019).

42. Coad, B. et al. Precommissural and postcommissural fornix microstructure in healthy aging and cognition. Brain Neurosci. Adv. https://doi.org/10.1177/2398212819899316 (2020).

43. Metzler-Baddeley, C., et al. Fornix white matter glia damage causes hippocampal gray matter damage during age-dependent limbic decline. In Scientific Reports, 1060 (Nature Publishing Group, 2019). 
44. Metzler-Baddeley, C., et al. Sex-specific effects of central adiposity and inflammatory markers on limbic microstructure. In NeuroImage, 793-803 (2019).

45. Zhang, H., Schneider, T., Wheeler-Kingshott, C. A. \& Alexander, D. C. NODDI: practical in vivo neurite orientation dispersion and density imaging of the human brain. Neuroimage 61, 1000-1016 (2012).

46. Cercignani, M. \& Alexander, D. C. Optimal acquisition schemes for in vivo quantitative magnetization transfer MRI. Magn. Reson. Med. 56, 803-810 (2006).

47. Eng, J., Ceckler, T. L. \& Balaban, R. S. Quantitative $1 \mathrm{H}$ magnetization transfer imaging in vivo. Magn. Reson. Med. 17, 304-314 (1991).

48. Henkelman, R. M., Stanisz, G. J. \& Graham, S. J. Magnetization transfer in MRI: a review. NMR Biomed. 14, 57-64 (2001).

49. Sled, J. G. Modelling and interpretation of magnetization transfer imaging in the brain. Neuroimage 182, 128-135 (2018).

50. Callaghan, M. F., Helms, G., Lutti, A., Mohammadi, S. \& Weiskopf, N. A general linear relaxometry model of R1 using imaging data. Magn. Reson. Med.. 73, 1309-1314 (2015).

51. Tuch, D. S. et al. High angular resolution diffusion imaging reveals intravoxel white matter fiber heterogeneity. Magn. Reson. Med. 48, 577-582 (2002).

52. Vogt, N. M. et al. Cortical microstructural alterations in mild cognitive impairment and Alzheimer's disease dementia. Cereb. Cortex 30(5), 2948-2960 (2019).

53. Fu, X., et al. Microstructural white matter alterations in mild cognitive impairment and Alzheimer's disease: study based on neurite orientation dispersion and density imaging (NODDI). Clin Neuroradiol (2019).

54. Slattery, C. F. et al. ApoE influences regional white-matter axonal density loss in Alzheimer's disease. Neurobiol. Aging 57, 8-17 (2017).

55. Colgan, N. et al. Application of neurite orientation dispersion and density imaging (NODDI) to a tau pathology model of Alzheimer's disease. Neuroimage 125, 739-744 (2016).

56. Ou, X., Sun, S. W., Liang, H. F., Song, S. K. \& Gochberg, D. F. Quantitative magnetization transfer measured pool-size ratio reflects optic nerve myelin content in ex vivo mice. Magn. Reson. Med. 61, 364-371 (2009).

57. Ou, X., Sun, S. W., Liang, H. F., Song, S. K. \& Gochberg, D. F. The MT pool size ratio and the DTI radial diffusivity may reflect the myelination in shiverer and control mice. NMR Biomed. 22, 480-487 (2009).

58. Samsonov, A. et al. Quantitative MR imaging of two-pool magnetisation transfer model parameters in myelin mutant shaking pup. Neuroimage 62, 1390-1398 (2012).

59. Kiefer, C. et al. Multi-parametric classification of Alzheimer's disease and mild cognitive impairment: the impact of quantitative magnetization transfer MR imaging. Neuroimage 48, 657-667 (2009).

60. Giulietti, G. et al. Quantitative magnetization transfer provides information complementary to grey matter atrophy in Alzheimer's disease brains. Neuroimage 59, 1114-1122 (2012).

61. Makovac, E. et al. Quantitative magnetization transfer of white matter tracts correlates with diffusion tensor imaging indices in predicting the conversion from mild cognitive impairment to Alzheimer's disease. J. Alzheimers Dis. 63, 561-575 (2018).

62. Tang, X. et al. Magnetic resonance imaging relaxation time in Alzheimer's disease. Brain Res. Bull. 140, 176-189 (2018).

63. Fischl, B. FreeSurfer. Neuroimage 62, 774-781 (2012).

64. Braak, H., Braak, E., Bohl, J. \& Bratzke, H. Evolution of Alzheimer's disease related cortical lesions. J. Neural Transm. Suppl. 54, 97-106 (1998).

65. IBM. SPSS Statistics, Version 20.0. (IBM Corp., Armonk, NY, 2011).

66. Nelson, H.E. The National Adult Reading Test-Revised (NART-R): Test

67. manual. (National Foundation for Educational Research-Nelson., Windsor, UK, 1991).

68. Benjamini, Y. \& Hochberg, Y. Controlling the false discovery rate: a practical and powerful approach to multiple testing. J. $R$. Stat. Soc. B 57, 289-300 (1995).

69. Yarnykh, V. L. et al. Iron-insensitive quantitative assessment of subcortical gray matter demyelination in multiple sclerosis using the macromolecular proton fraction. AJNR Am. J. Neuroradiol. 39, 618-625 (2018).

70. Wang, Y., van Gelderen, P., de Zwart, J. \& Duyn, J. B0 field dependence of MRI T1 relaxation in human brain. Neuroimage 213, $1-11(2020)$

71. Yin, C. et al. ApoE attenuates unresolvable inflammation by complex formation with activated C1q. Nat. Med. 25, 496-506 (2019).

72. de Chaves, E. P. \& Narayanaswami, V. Apolipoprotein E and cholesterol in aging and disease in the brain. Future Lipidol. 3, 505-530 (2008).

73. Gong, J. S. et al. Apolipoprotein E (ApoE) isoform-dependent lipid release from astrocytes prepared from human ApoE3 and ApoE4 knock-in mice. J. Biol. Chem. 277, 29919-29926 (2002).

74. Dansokho, C. \& Heneka, M. T. Neuroinflammatory responses in Alzheimer's disease. J. Neural Transm. (Vienna) 125, 771-779 (2018).

75. Sarlus, H. \& Heneka, M. T. Microglia in Alzheimer's disease. J. Clin. Invest. 127, 3240-3249 (2017).

76. Tejera, D. et al. Systemic inflammation impairs microglial A $\beta$ clearance through NLRP3 inflammasome. EMBO J. 38, e101064 (2019).

77. Amaral, D. G. \& Cowan, W. M. Subcortical afferents to the hippocampal formation in the monkey. J. Comp. Neurol. 189, 573-591 (1980).

78. Vogt, B. A., Pandya, D. N. \& Rosene, D. L. Cingulate cortex of the rhesus monkey. I Cytoarchitecture and thalamic afferents. J. Comp. Neurol. 262, 256-270 (1987).

79. Bubb, E. J., Kinnavane, L. \& Aggleton, J. P. Hippocampal-diencephalic-cingulate networks for memory and emotion: An anatomical guide. Brain Neurosci Adv 1, 1-20 (2017).

80. Johnson, S. C. et al. Amyloid burden and neural function in people at risk for Alzheimer's Disease. Neurobiol. Aging 35, 576-584 (2014).

81. Cacciaglia, R. et al. Effects of APOE- $\varepsilon 4$ allele load on brain morphology in a cohort of middle-aged healthy individuals with enriched genetic risk for Alzheimer's disease. Alzheimers Dement. 14, 902-912 (2018).

82. Chételat, G. et al. Larger temporal volume in elderly with high versus low beta-amyloid deposition. Brain 133, 3349-3358 (2010).

83. Harrison, N. A. et al. Quantitative magnetization transfer imaging as a biomarker for effects of systemic inflammation on the brain. Biol. Psychiatry 78, 49-57 (2015).

84. Dowell, N. G. et al. MRI of carriers of the apolipoprotein E e4 allele-evidence for structural differences in normal-appearing brain tissue in e4+ relative to e4- young adults. NMR Biomed. 26, 674-682 (2013).

85. Fouquet, M., Besson, F. L., Gonneaud, J., La Joie, R. \& Chételat, G. Imaging brain effects of APOE4 in cognitively normal individuals across the lifespan. Neuropsychol. Rev. 24, 290-299 (2014).

86. Willette, A. A. \& Kapogiannis, D. Does the brain shrink as the waist expands?. Ageing Res. Rev. 20, 86-97 (2015).

87. Feis, R. A. et al. Multimodal MRI of grey matter, white matter, and functional connectivity in cognitively healthy mutation carriers at risk for frontotemporal dementia and Alzheimer's disease. BMC Neurol. 19, 343 (2019).

88. Adluru, N. et al. White matter microstructure in late middle-age: Effects of apolipoprotein E4 and parental family history of Alzheimer's disease. Neuroimage Clin. 4, 730-742 (2014). 
89. Westlye, L. T., Reinvang, I., Rootwelt, H. \& Espeseth, T. Effects of APOE on brain white matter microstructure in healthy adults. Neurology 79, 1961-1969 (2012).

90. Dell'Acqua, F. et al. Tract based spatial statistic reveals no differences in white matter microstructural organization between carriers and non-carriers of the APOE $\varepsilon 4$ and $\varepsilon 2$ alleles in young healthy adolescents. J. Alzheimers Dis. 47, 977-984 (2015).

91. De Santis, S., Drakesmith, M., Bells, S., Assaf, Y. \& Jones, D. K. Why diffusion tensor MRI does well only some of the time: variance and covariance of white matter tissue microstructure attributes in the living human brain. Neuroimage 89, 35-44 (2014).

92. Timmers, I. et al. Assessing microstructural substrates of white matter abnormalities: a comparative study using DTI and NODDI. PLoS ONE 11, e0167884 (2016).

93. Mole, J. et al. Genetic risk of dementia modifies obesity effects on white matter myelin in cognitively healthy adults. Neurobiol. Aging 94, 298-310 (2020).

94. Fjell, A. M. et al. What is normal in normal aging? Effects of aging, amyloid and Alzheimer's disease on the cerebral cortex and the hippocampus. Prog. Neurobiol. 117, 20-40 (2014).

95. Billiet, T. et al. Age-related microstructural differences quantified using myelin water imaging and advanced diffusion MRI. Neurobiol. Aging 36, 2107-2121 (2015).

96. Nazeri, A. et al. Functional consequences of neurite orientation dispersion and density in humans across the adult lifespan. J. Neurosci. 35, 1753-1762 (2015).

97. Dickstein, D. L., Weaver, C. M., Luebke, J. I. \& Hof, P. R. Dendritic spine changes associated with normal aging. Neuroscience 251, 21-32 (2013)

98. Flood, D. G., Buell, S. J., Defiore, C. H., Horwitz, G. J. \& Coleman, P. D. Age-related dendritic growth in dentate gyrus of human brain is followed by regression in the "oldest old". Brain Res. 345, 366-368 (1985).

99. Flood, D. G., Buell, S. J., Horwitz, G. J. \& Coleman, P. D. Dendritic extent in human dentate gyrus granule cells in normal aging and senile dementia. Brain Res. 402, 205-216 (1987).

100. Rubinow, M. J., Drogos, L. L. \& Juraska, J. M. Age-related dendritic hypertrophy and sexual dimorphism in rat basolateral amygdala. Neurobiol. Aging 30, 137-146 (2009).

101. Whitaker, K. J. et al. Adolescence is associated with genomically patterned consolidation of the hubs of the human brain connectome. Proc. Natl. Acad. Sci. U.S.A. 113, 9105-9110 (2016).

102. Bourgeois, J. P. \& Rakic, P. Changes of synaptic density in the primary visual cortex of the macaque monkey from fetal to adult stage. J. Neurosci. 13, 2801-2820 (1993).

103. Petanjek, Z. et al. Extraordinary neoteny of synaptic spines in the human prefrontal cortex. Proc. Natl. Acad. Sci. U.S.A. 108, 13281-13286 (2011).

104. Karama, S. et al. Childhood cognitive ability accounts for associations between cognitive ability and brain cortical thickness in old age. Mol. Psychiatry 19, 555-559 (2014).

105. Sanchis-Segura, C. et al. Sex differences in gray matter volume: how many and how large are they really?. Biol. Sex Differ. 10, 32 (2019).

106. Tan, A., Ma, W., Vira, A., Marwha, D. \& Eliot, L. The hippocampus is not sexually-dimorphic: Meta-analysis of structural MRI volumes. Neuroimage 124, 350-366 (2016).

107. Kirsch, S. J., Jacobs, R. W., Butcher, L. L. \& Beatty, J. Prolongation of magnetic resonance T2 time in hippocampus of human patients marks the presence and severity of Alzheimer's disease. Neurosci. Lett. 134, 187-190 (1992).

108. Knight, M. J., Wearn, A., Coulthard, E. \& Kauppinen, R. A. T2 Relaxometry and diffusion tensor indices of the hippocampus and entorhinal cortex improve sensitivity and specificity of MRI to detect amnestic mild cognitive impairment and Alzheimer's disease dementia. J Magn. Reson. Imaging 49, 445-455 (2019).

109. Folstein, M., Folstein, S. \& McHugh, P. "Mini-mental state." A practical method for grading the cognitive state of patients for the clinician. J. Psychiatr. Res. 12, 189-198 (1975).

110. Kroenke, K., Spitzer, R. L. \& Williams, J. B. The PHQ-9: validity of a brief depression severity measure. J. Gen. Intern. Med. 16, 606-613 (2001).

111. Jones, D. K., Horsfield, M. A. \& Simmons, A. Optimal strategies for measuring diffusion in anisotropic systems by magnetic resonance imaging. Magn. Reson. Med. 42, 515-525 (1999).

112. Jezzard, P. \& Balaban, R. S. Correction for geometric distortion in echo planar images from B0 field variations. Magn. Reson. Med. 34, 65-73 (1995).

113. Leemans, A. \& Jones, D. K. The B-matrix must be rotated when correcting for subject motion in DTI data. Magn. Reson. Med. 61, 1336-1349 (2009).

114. Leemans, A., Jeurissen, B., Sijbers, J. \& Jnes, D.K. ExploreDTI: a graphical toolbox for processing, analyzing, and visualizing diffusion MR data. In 17th Annual Meeting of Intl Soc Mag Reson Med 3537 (Hawaii, USA., 2009).

115. Irfanoglu, M. O., Walker, L., Sarlls, J., Marenco, S. \& Pierpaoli, C. Effects of image distortions originating from susceptibility variations and concomitant fields on diffusion MRI tractography results. Neuroimage 61, 275-288 (2012).

116. Daducci, A. et al. Accelerated microstructure imaging via convex optimization (AMICO) from diffusion MRI data. Neuroimage 105, 32-44 (2015)

117. Klein, S., Staring, M., Murphy, K., Viergever, M. A. \& Pluim, J. P. elastix: a toolbox for intensity-based medical image registration. IEEE Trans. Med. Imaging 29, 196-205 (2010).

118. Ramani, A., Dalton, C., Miller, D. H., Tofts, P. S. \& Barker, G. J. Precise estimate of fundamental in-vivo MT parameters in human brain in clinically feasible times. Magn. Reson. Imaging 20, 721-731 (2002).

119. Jenkinson, M., Beckmann, C. F., Behrens, T. E., Woolrich, M. W. \& Smith, S. M. FSL. Neuroimage 62, 782-790 (2012).

120. Smith, S. M. et al. Advances in functional and structural MR image analysis and implementation as FSL. Neuroimage 23(Suppl 1), S208-219 (2004).

121. Dale, A. M., Fischl, B. \& Sereno, M. I. Cortical surface-based analysis I. Segmentation and surface reconstruction. Neuroimage 9, 179-194 (1999).

122. Desikan, R. S. et al. An automated labeling system for subdividing the human cerebral cortex on MRI scans into gyral based regions of interest. Neuroimage 31, 968-980 (2006).

123. Fischl, B. \& Dale, A. M. Measuring the thickness of the human cerebral cortex from magnetic resonance images. Proc. Natl. Acad. Sci. U.S.A. 97, 11050-11055 (2000)

124. Fischl, B. et al. Whole brain segmentation: automated labeling of neuroanatomical structures in the human brain. Neuron 33, 341-355 (2002)

125. Johnson, A. R., Milner, J. J. \& Makowski, L. The inflammation highway: metabolism accelerates inflammatory traffic in obesity. Immunol. Rev. 249, 218-238 (2012).

126. Cohen, J. Statistical Power Analysis for the Behavioral Sciences (Lawrence Earlbaum Associates, NJ, 1988).

\section{Acknowledgements}

This research was funded by a Research Fellowship awarded to CMB from the Alzheimer's Society and the BRACE Alzheimer's Charity (grant ref: 208). JPA is supported by the Wellcome Trust (grant 103722/Z14/Z). We would like to thank Erika Leonaviciute, Peter Hobden and Sonya Foley-Bozorgzad for their assistance with MRI 
data acquisition and Rosie Dwyer, Samantha Collins, Abbie Stark, and Emma Blenkinsop for their assistance with the collection and scoring of the cognitive and health data. We also would like to thank Rhodri Thomas for his assistance with the APOE genotyping of the saliva samples.

\section{Author contributions}

C.M.B.: conceptualization, methodology, formal analysis, writing-original draft preparation, writing-review and editing, visualization, funding acquisition; J.P.M.: investigation, formal analysis, data curation, project administration; R.S., E.K.: Resources; F.F., J.E.: Software; J.A.: reviewing and editing.

\section{Competing interests}

The authors declare no competing interests.

\section{Additional information}

Supplementary information is available for this paper at https://doi.org/10.1038/s41598-020-75992-9.

Correspondence and requests for materials should be addressed to C.M.-B.

Reprints and permissions information is available at www.nature.com/reprints.

Publisher's note Springer Nature remains neutral with regard to jurisdictional claims in published maps and institutional affiliations.

(c) (1) Open Access This article is licensed under a Creative Commons Attribution 4.0 International License, which permits use, sharing, adaptation, distribution and reproduction in any medium or format, as long as you give appropriate credit to the original author(s) and the source, provide a link to the Creative Commons licence, and indicate if changes were made. The images or other third party material in this article are included in the article's Creative Commons licence, unless indicated otherwise in a credit line to the material. If material is not included in the article's Creative Commons licence and your intended use is not permitted by statutory regulation or exceeds the permitted use, you will need to obtain permission directly from the copyright holder. To view a copy of this licence, visit http://creativecommons.org/licenses/by/4.0/.

(C) The Author(s) 2020 